\title{
Limbic and cortical control of phonation for speech in response to a public speech preparation stressor
}

\author{
Maria Dietrich $^{1}$ (D) Richard D. Andreatta ${ }^{2} \cdot$ Yang Jiang $^{3} \cdot$ Joseph C. Stemple ${ }^{2}$
}

Published online: 2 May 2019

(C) The Author(s) 2019

\begin{abstract}
Knowledge on brain networks subserving vocalization in vocally healthy individuals under various task conditions is scarce but paramount to understand voice disorders. The aims of our study were to determine (1) the effect of social-evaluative stress on the central neural control of phonation underlying speech production; and (2) the neural signature, personality profile, and aerodynamic vocal function in relation to salivary cortisol responses. Thirteen vocally healthy females underwent an event-related sparse-sampling fMRI protocol consisting of voiced and whispered sentence productions with and without exposure to the social-evaluative stressor public speaking anticipation. Participants completed a personality questionnaire, rating scales of negative emotional state, and provided salivary cortisol samples. In the total sample, the task contrast of voiced productions revealed that stressor exposure resulted in a peak activation in the right caudate with concomitant deactivations in the bilateral pgACC and aMCC, and right IFG, BA 9, BA 10, insula, putamen, and thalamus. There were individual differences in stressor-induced brain activations as a function of stress reactivity with greater cortisol reactivity linked with lower laryngeal motor cortex activity and lower scores on aspects of extraversion. Our data confirm that stress alters the phonatory control for speech production through limbic-motor interactions. The findings support the Trait Theory of Voice Disorders (Roy and Bless 2000) and help provide critical insights to the study of voice disorders such as primary muscle tension dysphonia.
\end{abstract}

Keywords Functional MRI $\cdot$ Limbic $\cdot$ Stress $\cdot$ Larynx $\cdot$ Voice disorders $\cdot$ Muscle tension dysphonia

Maria Dietrich

dietrichm@health.missouri.edu

Richard D. Andreatta

richard.andreatta@uky.edu

Yang Jiang

yjiang@uky.edu

Joseph C. Stemple

joseph.stemple@uky.edu

1 Department of Speech, Language and Hearing Sciences, University of Missouri, 308 Lewis Hall, Columbia, MO 65211, USA

2 Department of Communication Sciences and Disorders, University of Kentucky, 120 Wethington Bldg, 900 S. Limestone, Lexington, KY 40536, USA

3 Department of Behavioral Science, University of Kentucky, 113 Medical Behavioral Science Building, Lexington, KY 40536, USA

\section{Introduction}

Phonatory control for speech operates under fluctuating cognitive and emotional states. While humans exert volitional control over phonation underlying speech production via primarily direct corticomotoneuronal pathways (Jürgens 2002), the direct or indirect impact of emotion on phonation for speech remains ambiguous at best. Primary vocal motor areas include the laryngeal motor cortex (LMC), premotor cortex, supplementary motor area (SMA), and lobule VI of the cerebellum, whereas secondary areas include the cingulate motor area, the ventral tier nuclei of the thalamus, the putamen, frontal operculum, and the anterior insula (Brown et al. 2009). In contrast to humans, primates regulate vocalizations through a limbic vocal motor pathway that relies on the anterior cingulate cortex (ACC) and periaqueductal gray (PAG) (Jürgens 2002). In humans, the analogous limbic vocal motor pathway plays a role in non-speech emotional vocalizations, such as crying or laughing (Ludlow 2005). While limbic activations 
have been reported during vocalizations for the purpose of speech (Haslinger et al. 2005; Loucks et al. 2007; Olthoff et al. 2008; Schulz et al. 2005), it has not been unequivocally determined whether these are epiphenomenal or essential to human phonatory control and speech production (Ludlow et al. 2008). A deeper understanding of the contribution of limbic activity during voice production such as the effects of emotion and psychological stress is warranted given the potentially profound modulatory effects of voice-related sensorimotor processing that may help to explain the origins of selected classes of voice disorders (Dietrich et al. 2012).

The Trait Theory of Voice Disorders (Roy and Bless 2000) has been proposed as a means for describing the potential clinical relevance of the limbic system and its interference role with voice production. The central hypothesis is that functional pathways between the limbic system and laryngeal sensorimotor control regions may help to explain individual differences in dispositional-related laryngeal motor behavior and the risk for voice disorders. For example, a predisposition to react with behavioral inhibition to punishment, novelty, or threat, assumed to be greater in individuals with introverted than extroverted traits, would interrupt or halt motor cortical activity and result in heightened and/or disorganized peripheral laryngeal muscle activity. Such heightened and/or disorganized muscle activity is thought to underlie complaints of perceived vocal effort and strain, which are, coincidently, hallmark symptoms of primary muscle tension dysphonia.

Data from connectivity analyses for the LMC may be compatible with the Trait Theory. Structural connectivity between the LMC and the middle cingulate cortex (MCC) has been shown and to a lesser extent connectivity with the dorsolateral prefrontal cortex (dlPFC) and midbrain (Simonyan et al. 2009). Additionally, functional connectivity data for the LMC have indicated that connectivity with the ACC and MCC was negative (weakened) during syllable productions, but positive connectivity would be plausible for complex speech, and that positive (enhanced) connections were found for the dIPFC, ventrolateral PFC (vlPFC), and midbrain. In the limbic system, the ACC integrates emotional and motivational states into cognitive processes, with its output influencing motor control for voice or vocal control (Paus 2001; Paus et al. 1993). Further, the ACC, amygdala, and septohippocampal system are neural correlates of behavioral inhibition (McNaughton and Corr 2004).

The clinical implications for this study are that in some patients with voice disorders, acute or chronic life stress accompanies the occurrence of primary muscle tension dysphonia, a voice disorder in the absence of vocal fold lesions, vocal fold paralysis, or a diagnosed psychological disorder (Dietrich et al. 2008; Roy 2003; Verdolini et al. 2006). In contrast, secondary muscle tension dysphonia occurs when vocal fold lesions or neurological or psychological etiologies are the primary source of the voice disorder and lead to compensatory muscular responses (Verdolini et al. 2006). The limbic system is involved in the initial processing of information and regulation of the stress response (Dedovic et al. 2009b) and participates in vocal control. Consequently, studying the relation between stress and the limbic and cortical control of phonation for speech production is a promising avenue to improve our understanding of individual differences in vocal control and the ever-present risks for the emergence of stress-related voice disorders (Dietrich et al. 2012).

Only three studies have been completed to date that explored the neurobiological bases of primary muscle tension dysphonia; (1) a cross-sectional study (Kryshtopava et al. 2017), (2) a case study pre- and post-successful laryngeal reposturing treatment (Roy et al. 2017), and (3) a study that explored two patients with muscle tension aphonia pre- and post-successful circumlaryngeal therapy (Spengler et al. 2017). The case studies, in particular, supported the hypothesis that dysfunctional limbic-motor interactions may underlie muscle tension laryngeal dysphonias, consistent with the Trait Theory. Still absent in the literature is detailed knowledge on cortical and subcortical brain networks subserving vocalization under various task conditions in vocally healthy individuals (Ludlow 2005; Simonyan et al. 2009). As such, we are lacking studies that have collected both physiological and brain responses to stress and individual reactivity during overt and covert voice production. This particular research direction, informed by the Trait Theory, is described in the Psychobiological Framework for Studying Psychological Stress and its Relation to Voice Disorders (Dietrich and Verdolini Abbott 2008; Helou 2014). This framework has been successfully used to study peripheral vocal function during a stress reactivity protocol (Dietrich and Verdolini Abbott 2012, 2014; Helou 2014). Integrating stress reactivity perturbations and fMRI protocols to study voice and speech production is novel and necessitates a feasibility and pilot study. Our central aim in this report was to provide initial data that imposed stress alters phonatory control for speech, which would support a key claim of the Trait Theory that individuals who score low on extroversion are prone to interrupt or halt motor cortical activity in response to novelty or threat with further implications for peripheral laryngeal function.

The objectives of our feasibility and pilot study were to determine (1) the effect of social-evaluative stress on the central neural control of phonation underlying speech production; and (2) the neural signature, personality profile, and aerodynamic vocal function of individuals in relation to their salivary cortisol responses. Based on the Trait Theory, we hypothesized that stressor exposure will alter laryngeal motor and premotor control, especially in those individuals who are stress responders. Based on previous fMRI research using a "speech preparation" activity as a stressor (Lorberbaum et al. 2004; Wager et al. 2009) and the Montreal Stress Imaging Task (Pruessner et al. 2008), we expected the following 
stressor-induced neural events: activations in the primary motor cortex (M1), premotor cortex, pre-SMA, middle frontal gyrus (MFG), ACC, MCC, insula, HC, thalamus, caudate, $\mathrm{PAG}$, and cerebellum; deactivations in the sensorimotor cortex (S1, M1), ventromedial PFC (vmPFC), OFC, vlPFC, posterior cingulate cortex (PCC), superior temporal gyrus (STG), insula, putamen, amygdala, HC, and cerebellum. Furthermore, we expected that individuals who would be more stress reactive based on cortisol would be characterized by lower self-esteem (Dickerson and Kemeny 2004), lower extroversion (Dietrich and Verdolini Abbott 2014), and greater laryngeal airway resistance than the non-responder group. Greater laryngeal airway resistance is a proxy measure of increased muscle tension in the laryngeal system of vocalizing humans (Hillman et al. 1989).

\section{Materials and methods}

\section{Participants}

Participants in the study were 13 females with a mean age of 21.7 years $(S D=4.6$, range: $18-35$ years). All participants were right-handed, native speakers of English, and in good physical, mental, and vocal health. Considering that this was a feasibility and pilot study, a pre-screening protocol was used to pre-organize the participant pool on the personality trait of stress reactivity. The goal was to balance the number of participants who scored below and above the norm on the 12item true/false Stress Reaction scale from the Multidimensional Personality Questionnaire-Brief Form (MPQ-BF; Patrick et al. 2002). The pre-screening protocol also included the Social Closeness scale to disguise the study's focus on stress reactivity. The median $T$ score on Stress Reaction was 40 with seven participants who scored at or below the median $(T=34-40)$ and six above the median $(T=48-66)$. In other words, there was a mix of participants ranging from $1.5 S D$ below the norm to $1.5 S D$ above the norm on the trait of Stress Reaction.

General exclusion criteria were smoking; upper respiratory infection, allergies or reflux disease affecting voice; hearing loss; pulmonary, cardiac, neurological, or endocrine diseases; current psychiatric or psychological treatment; and body mass index in the obese range (BMI $>30$, not fitting into the scanner). Voice-specific exclusion criteria were a current or lifetime history of a voice disorder, vocal pathology, laryngeal trauma or neck surgery, previous voice therapy, professional singing or voice training. Vocal health status was determined by laryngeal videostroboscopy and clinically standard auditory-perceptual evaluation of voice production. Participants who did not achieve mid-membranous vocal fold closure during modal pitch and comfortable loudness were excluded. Two of the authors are certified speech-language pathologists (MD, JCS) and reviewed the exams independently. Complete agreement was required on a participant's vocal health status before inclusion. The study was approved by the University of Kentucky Institutional Review Board as a deception study. All participants provided informed consent and were compensated monetarily for their time. Participants were fully debriefed about the true focus and nature of the research before exiting the study.

\section{Functional MRI paradigm}

An event-related sparse-sampling design was used to study voice and speech production, capitalizing on the delayed task-related hemodynamic response in the brain (Perrachione and Ghosh 2013). The TR was seven seconds split into three seconds for volume acquisition and a silent delay of four seconds for speech production during which the scanner gradients were turned off (jittered 3.5-4.5 s). Meanwhile, the duration of volume acquisition was also used for the projection of task instructions onto a translucent screen (Silent Vision SV6011 LCD, Avotec Inc., Stuart, FL) using E-Prime software (Psychology Software Tools Inc., Pittsburgh, PA). All participants wore MRI-compatible headphones with a microphone to communicate with the experimenters (Resonance Technology Inc., Northridge, CA).

The tasks included 30 trials of voiced sentence reading (Voice), 30 trials of whispered sentence reading (Whisper), and 40 trials of rest (fixation cross) distributed across two runs per experimental condition (No Stress vs. Stress). The trials for the voiced versus whispered task conditions were presented in a pseudo-randomized order within each run with the sentence stimuli repeated across trials. The stimuli were selected short sentences from the Consensus Auditory Perceptual Evaluation of Voice (Kempster et al. 2009) that were biased with voiced sounds (obligatory vocal fold vibration required for production): The blue spot is on the key again (production of every vowel sound in English), We were away a year ago (all voiced), We eat eggs every Easter (vowel onsets). Phonemes and syllables have been used in past fMRI research aimed at localizing the LMC (Brown et al. 2008; Simonyan et al. 2009). Here we chose sentences to capture more complex and naturalistic speech beyond simple laryngeal tasks (e.g. /ihi/). Further, the task contrast of Voice versus Whisper was designed to isolate phonation from articulatory aspects of speech production. The Voice task condition completely engaged the larynx, requiring the participant to use the full range of neuromuscular control to achieve medial compression and stiffness regulation of the vocal folds. On the other hand, the Whisper task condition required minimal and incidental movement of the vocal folds, relying instead on airflow turbulence through a narrowed glottis to generate acoustical sources of energy (Konnai et al. 2017). The same sentences were produced in both experimental conditions (No 
Stress vs. Stress) so that articulatory activity and language content were equivalent across task conditions. The expected net result would be the task-related cortical activity underlying laryngeal control for speech.

\section{Experimental stress induction}

After our baseline conditions, social-evaluative stress was imposed using a modified public speaking stressor script extracted from the Trier Social Stress Test (Kirschbaum et al. 1993). Participants were told that they had to give a five-minute impromptu "speech" about why they are the best candidate for a position in a law firm. The script was modified such that it did not include a preparation phase and ended with the caveat that for control purposes there was a small chance that the prepared speech would not be delivered. Participants were then prompted to read the stimuli again while mentally preparing for their speeches. The scanner runs started and ended with visual cues indicating that the speech may start at any moment. Elements of time pressure, uncertainty, and social evaluation (participants were told that three experimenters would be observing their performances) were used to uphold the psychological tension during the stressor task period. None of the participants actually delivered a speech.

\section{Measurements}

\section{Endocrine measures}

A key limitation of many stress studies is a failure to validate the stress response with cortisol measures (Dedovic et al. 2009a), an objective marker of the biological stress response (Dickerson and Kemeny 2004). Seven samples were collected with Salimetrics Oral Swabs (Salimetrics, State College, PA) in approximately 10-minute intervals starting 45-60 min before the onset of the stressor (first sample immediately before mock scanner training) until 40-50 min post stressor (Fig. 1). Cortisol peaks 20-40 min post-stressor and all cortisol samples were collected in the late afternoon/early evening (4:007:00 p.m.) to best capture a consistent cortisol response (Dickerson and Kemeny 2004). When participants were in the MRI scanner, saliva oral swab tubes were placed in the participant's hand between runs. The routine of placing the oral swab in their mouth and back in the tube was practiced in the mock scanner before actual data collection.

\section{Psychometric measures of personality}

The MPQ-BF was chosen for the pre-screening because it contains a separate scale to measure Stress Reaction (dimension Negative Emotionality). For a full personality assessment, participants completed the NEO-Personality InventoryRevised (NEO-PI-R; Costa and McCrae 1992) during screening. The 240-item NEO-PI-R reflects the integrative trait taxonomy with the factors of Neuroticism, Extraversion, Openness, Agreeableness, and Conscientiousness with each factor comprising several subscales. Each question was answered on a 5-point Likert scale ranging from strongly disagree to strongly agree. Participants also completed the BIS/BAS personality scales (Carver and White 1994) and the Rosenberg self-esteem scale (Rosenberg 1989) during screening. The 24-item BIS/BAS scales (7 items BIS) capture the behavioral motivational systems behavioral inhibition and behavioral activation. Questions were answered on a 4-point Likert scale ranging from very true for me to very false for me. Questions on the 10-item Rosenberg scale were answered on a 4-point Likert scale ranging from strongly agree to strongly disagree. A low Rosenberg score has been shown to be linked to greater cortisol responsiveness during social-evaluative stressor exposure (Dickerson and Kemeny 2004).

\section{Psychoemotional state measures}

Throughout the experiment participants provided ratings of their emotional state using selected items from the Positive and Negative Affect Scale-Expanded Form (PANAS-X; Watson and Clark 1994). Participants rated the six items of the basic negative emotion scale for fear on a 5-point Likert scale ranging from very slightly or not at all to extremely after training in the mock scanner, via intercom after the baseline phase and stress phase, and in writing for the recovery phase. After participants left the scanner, they also completed the 30item true/false Personal Report of Confidence as a Speaker (PRCS; Paul 1966) to capture the degree of fear of public speaking they experienced during stressor exposure.

\section{Vocal function measures}

During the screening, participants completed the 30-item Voice Handicap Index (Jacobson et al. 1997) using a 5-point Likert scale from never to always, which assessed the impact that a voice disorder may have on perceived quality of life in functional, physical, and emotional domains. Immediately before the MRI session, data on aerodynamic vocal function were collected using the automated computer-based Phonatory Aerodynamic System (PAS, model 6600, KayPentax, Montvale, NJ). The voicing efficiency protocol, consisting of a string of five plosive consonant-vowel syllables (/pa/), was used to determine mean peak subglottal pressures $\left(\mathrm{cm} \mathrm{H}_{2} \mathrm{O}\right)$, mean airflow during voicing (L/s), and laryngeal airway resistance $\left(\mathrm{R}_{\text {law }}=\mathrm{cm} \mathrm{H} \mathrm{H}_{2} \mathrm{O} /[\mathrm{L} / \mathrm{s}]\right)$. $\mathrm{R}_{\text {law }}$ is considered a proxy measure for vocal effort and laryngeal muscle activity. The implications of an elevated $\mathrm{R}_{\text {law }}$ would be an increased risk for excessive vocal effort and fatigue, a commonplace outcome in occupational voice users (Roy et al. 2004). 


\section{Procedures}

Figure 1 shows the timeline of the experimental procedures. Prior to the experiment, participants were asked to abstain from alcohol (12 h), exercise (on the day of the experiment), a large meal $(2 \mathrm{~h})$, and caffeine $(3 \mathrm{~h})$ (MacArthur Research Network on Socioeconomic Status and Health 2000; Shapiro et al. 1996). Data on voicing efficiency were collected first. Participants were then escorted to the University of Kentucky's MRI \& Spectroscopy Center's mock scanner where they were trained on the tasks and familiarized with the scanning environment. After training, participants were set up in the research MRI scanner. During the No Stress condition (first two runs), participants read short sentences in voiced and whispered manners or rested quietly (conditions Voice No Stress [VoiceNS], Whisper No Stress [WhisperNS]). After that, participants were told for the first time that they had to give a 5-min impromptu speech while being observed and evaluated by a panel of three external individuals. During the Stress condition that followed (third and fourth runs), participants were prompted to again read sentences in voiced and whispered manners, however this time, while waiting for their prompt to start their speech (conditions Voice Stress [Voices], Whisper Stress [WhisperS]). After the Stress runs, the "speech" delivery was waived and participants were fully debriefed as to the actual purpose of the research. Participants provided ratings of their negative emotional state using the PANAS-X after training in the mock scanner, after the No Stress condition, after the Stress condition in the scanner, and lastly after the recovery period outside the scanner followed by completion of the PRCS. Seven salivary cortisol samples were collected with oral swabs in approximately 10-min intervals throughout the experiment.

\section{FMRI data acquisition}

The fMRI data were acquired on a Siemens Magnetom Trio Tim 3T scanner (Siemens, Erlangen, Germany) equipped with a 32-channel head coil. Data from four functional runs were collected using T-2*-weighted gradient echo echo-planar imaging (EPI) scans (acquisition parameters: $\mathrm{TR}=7.0 \mathrm{~s}$, $\mathrm{TA}=$ $3.0 \mathrm{~s}$, delay $=4.0 \mathrm{~s}, \mathrm{TE}=30 \mathrm{~ms}$, flip angle $=81^{\circ}, \mathrm{FoV}=$ $224 \mathrm{~mm} \times 224 \mathrm{~mm}, 64 \times 64$ matrix, slice thickness $=$ $3.5 \mathrm{~mm}$, voxel resolution $=3.5 \mathrm{~mm} \times 3.5 \mathrm{~mm} \times 3.5 \mathrm{~mm}$, band width $=2056 \mathrm{~Hz} / \mathrm{Px}, 40$ interleaved axial slices providing whole-brain coverage, number of volumes $=224$ ). A wholebrain high-resolution anatomical volume was acquired using a T-1weighted magnetization-prepared rapid gradient echo (MPRAGE) sequence (acquisition parameters: TR = $2100 \mathrm{~ms}, \mathrm{TE}=2.93 \mathrm{~ms}, \mathrm{TI}=1100 \mathrm{~ms}$, flip angle $=12^{\circ}$, FoV $=224 \mathrm{~mm} \times 256 \mathrm{~mm}$, voxel resolution $=1 \mathrm{~mm}$ isotropic voxels). At the beginning of each run, three TRs with no data were discarded to allow for stabilization of longitudinal magnetization.

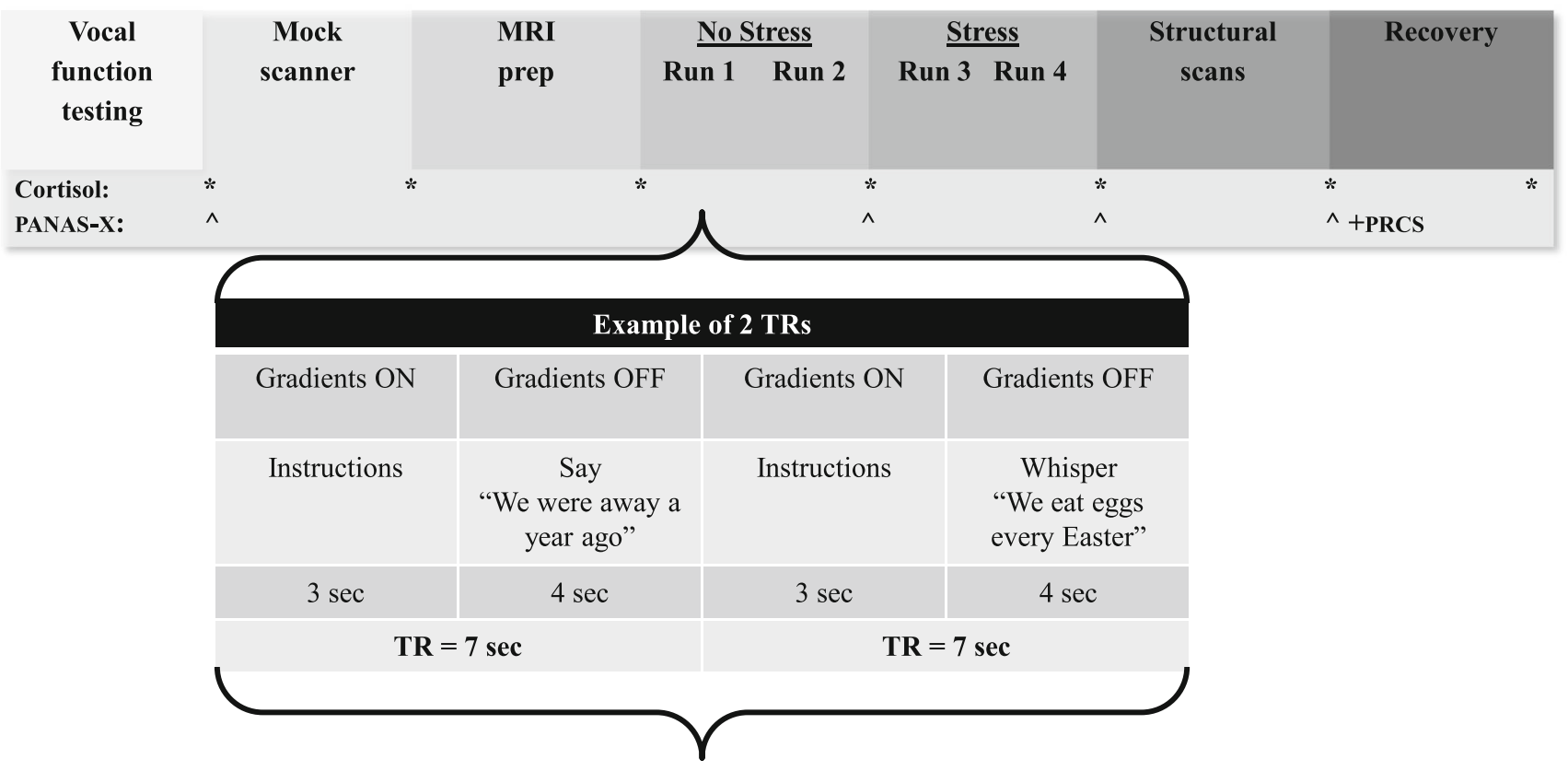

Fig. 1 Timeline of procedures along with outcome measures and an example of the event-related sparse-sampling design. PANAS-X = Positive and Negative Affect Scales-Expanded Form; PRCS = Personal Report of Confidence as a Speaker 


\section{Data analysis}

\section{Data analysis of endocrine measures}

Salivary cortisol samples were stored in a $-80^{\circ} \mathrm{C}$ freezer and then analyzed in duplicate at the University of Kentucky Clinical Research Development and Operations Center Core Biochemical Analysis Laboratory (Salivary Cortisol Kit 13002, Salimetrics, State College, PA). A participant was a stress responder when the cortisol area under the curve with reference to increase $\left(\mathrm{AUC}_{\mathrm{I}}\right)$ (Pruessner et al. 2003) was positive. Participants were categorized as non-responders when $\mathrm{AUC}_{\mathrm{I}}$ levels were zero or negative. Personality characteristics and aerodynamic vocal function were correlated with these salivary responses. In addition, non-responders and stress responders were descriptively compared on personality characteristics and aerodynamic vocal function.

\section{Data analysis of psychometric, psychoemotional, and vocal function measures}

For the psychometric measures, descriptive statistics were determined for the two MPQ-BF subscales Stress Reaction and Social Closeness, the five factors of the NEO-PI-R, the BIS subscale, and the Rosenberg scale. The scores on fear ratings (PANAS-X) for the total group were statistically compared across conditions (No Stress, Stress, recovery) using a within-subjects ANOVA. The $p$ value was set at .05 . The statistical analyses were performed with SPSS Statistics for Windows, Version 21.0 (IBM Corp., Armonk, NY). Descriptive statistics were determined for fear of public speaking (PRCS). Voicing efficiency data were analyzed by averaging the middle three productions of $/ \mathrm{pa} /$ from a set of five to determine values for subglottal pressure, airflow, and $\mathrm{R}_{\text {law }}$ following recommendations by Solomon and Helou (2013).

\section{FMRI data analysis}

Preprocessing, analysis, and presentation of fMRI data were completed with Analysis of Functional NeuroImages software, version AFNI_2011_12_21_1014 (http://afni.nimh. nih.gov/afni) (Cox 1996) with the exception of the preparation of field maps and the subsequent correction of $\mathrm{B} 0$ distortions in the EPI data, which was carried out using FSL version 5.0.8 (FMRIB's Software Library, www.fmrib.ox.ac.uk/fsl) (Jenkinson et al. 2012).

Preprocessing Data were converted from DICOM format to FSL NIfTI format using MRIConvert (http://lcni.uoregon.edu/ downloads/mriconvert/mriconvert-and-mcverter) and also from DICOM to AFNI format using to3d. Field maps were prepared using the FSL tool $f$ sl prepare fieldmap. The FSL command line tool fugue was then used to unwarp B0 distortions in the EPI data (EPI files were converted in AFNI to apply the correct volume acquisition timing for sparse sampling, then converted to NIfTI format using $3 d A F N t o N I F T I$ ). The processing pipeline was generated using uber_subject.py (version 0.39) that ran the afni_proc. py super-script (Cox 2012). In a single transformation, the EPI and high-resolution anatomical images were co-registered, normalized to MNI template space (MNI Avg152, T1 1x1x1 $\mathrm{mm}$ ) using non-linear warping, and the data were motion corrected using cubic polynomial interpolation. The reference volume for volume registration (realignment) was the last volume of the last run because the anatomical dataset was acquired after the EPI. We applied a $4.0 \mathrm{~mm}$ FWHM Gaussian filter to smooth the data (on top of the existing blur) and obtained estimates of the final blur of the data (approximately $6 \mathrm{~mm}$ ). A moderate smoothing value was chosen to balance the signal-to-noise ratio. The final blur estimates were averaged across participants and used in $3 d$ ClustSim to estimate the probability of false-positive clusters. Finally, a brain mask was created from the EPI data (dilate 1 voxel) and each voxel was scaled to a mean of 100 for units in percent signal change. Slice timing correction was not applied to the EPI data because a sparse-sampling design was used for volume acquisition and thus, data acquisition was discontinuous during the TR.

Model design and estimation Regression analysis was completed using a fixed shaped regression using AFNI BLOCK, which convolves an incomplete gamma function with a boxcar function, where each has a height of 1 . The BLOCK curve lasts about $15.8 \mathrm{~s}$ longer than the stimulus duration ( $3.5 \mathrm{~s})$. Each beta weight represents the peak magnitude of the response to the entire stimulus block. First, we examined the main effects of each condition No Stress (NS) and Stress (S) condition: VoiceNS, VoiceS, WhisperNS, WhisperS. Then, the program uber_ttest.py (version 1.1) was used at the group level to test the following contrasts: VoiceS-VoiceNS, WhisperS-WhisperNS, WhisperS-VoiceS, and WhisperNSVoiceNS. Clusters were corrected for multiple comparisons to achieve a family-wise error (FWE) rate of $p<.05$.

\section{ROI data analysis}

Anatomical regions of interest (ROI) were defined a priori and primarily informed by evidence about primary and secondary vocal areas (Brown et al. 2009) as well as the functional connectome of speech control under consideration of laterality (Fuertinger et al. 2015; Simonyan and Horwitz 2011) and corticolimbic and limbic regions involved in stress responses (Dedovic et al. 2009b; Lorberbaum et al. 2004; Wager et al. 2009). Masks were created using the AFNI adaptions of the Eickhoff-Zilles maximum probability maps (areas $4 p$ and 6 ) and macro label maps (postcentral gyrus, SMA, MFG, ACC, 
MCC, insula, thalamus, putamen, amygdala, HC, and lobule VI of the cerebellum) (Eickhoff et al. 2005). These maps were also used to label anatomical regions for the whole brain analyses. In addition, a bilateral ROI mask for the PAG with the stereotaxic Talairach space coordinates $\mathrm{x}$-axis -8 to +8 , $\mathrm{y}$ axis -26 to -33 , and $z$-axis -4 to -16 was drawn manually using AFNI.

BOLD percent signal changes were extracted for each ROI for the Voice No Stress and Voice Stress conditions and were correlated with cortisol data using Spearman's rho to gain insight about individual differences in BOLD reactivity as a function of perceived stress. In a second step, BOLD data that correlated significantly with $\mathrm{AUC}_{\mathrm{I}}$ values were correlated with personality characteristics and aerodynamic vocal function. Because of the exploratory nature of investigating individual differences, the $p$ value was $<.1$ (two-tailed).

\section{Results}

\section{Salivary cortisol}

Calculations of $\mathrm{AUC}_{\mathrm{I}}$ for salivary cortisol were based on samples collected immediately before the onset of the stressor until approximately $50 \mathrm{~min}$ after the stressor event. The first three cortisol samples were not included in our analysis because of possible contamination by the participants' experience in the mock scanner from which they would have recovered before stressor induction. Four participants showed positive $\mathrm{AUC}_{\mathrm{I}}$ values (stress responders) while nine showed negative $\mathrm{AUC}_{\mathrm{I}}$ values (non-responders). Figure 2 shows cortisol data for the stress responders and non-responders over time.

\section{Psychometric measures}

Table 1 shows the personality characteristics of the total sample (including significant correlations with $\mathrm{AUC}_{\mathrm{I}}$ ) as well as those of stress responders and non-responders. Stress responders scored higher than non-responders on all scales of Neuroticism and Openness (except Ideas and Values), lower on all scales of Extraversion (except for Excitement-Seeking) and Conscientiousness, and mixed on Agreeableness (lower on Trust, Compliance, Tender-Mindedness and higher on Straightforwardedness, Altruism, Modesty). Further, stress responders had lower mean scores on behavioral inhibition and self-esteem. Significant correlations with $\mathrm{AUC}_{\mathrm{I}}$ values in the total sample were found for Extraversion-Activity (-.58, $p=.037)$, Agreeableness-Modesty (.51, $p=.077)$, and Conscientiousness-Achievement Striving $(-.65, p=.016)$.

\section{Psychoemotional stress reactivity}

The scores for the fear subscale (PANAS-X) in the total sample were compared among the No Stress $(M=7.0, S D=0.9)$, Stress $(M=13.9, S D=3.7)$, and recovery conditions $(M=6.6, S D=$ $0.8)$. The main effect of condition on ratings of fear was significant: $F(2,24)=46.46, p<.001$, partial $\eta^{2}=0.80$. Planned comparisons revealed that ratings for the Stress condition were significantly greater than ratings for either the No Stress or recovery conditions, $p<.001$. The ratings for the baseline and recovery phases were not significantly different. Figure 3 shows the results. The mean Personal Report of Confidence as a Speaker (PRCS) score in the total sample was $15.7(S D=$ 6.5). Stress responders had a mean of $20.0(S D=1.4)$ and non-responders a mean of $13.8(S D=7.1)$ (possible maximum score of 30 indicating greater fear of public speaking).
Fig. 2 Time course of salivary cortisol levels in stress responders and non-responders pre and post stress induction. Time zero indicates the cortisol sample immediately before experimental stress induction. Cortisol peaks 20-40 min post stressor. Practice in the mock scanner elicited reactivity in some participants. Therefore, area under the curve with respect to increase $\left(\mathrm{AUC}_{\mathrm{I}}\right)$ was calculated using the last four samples

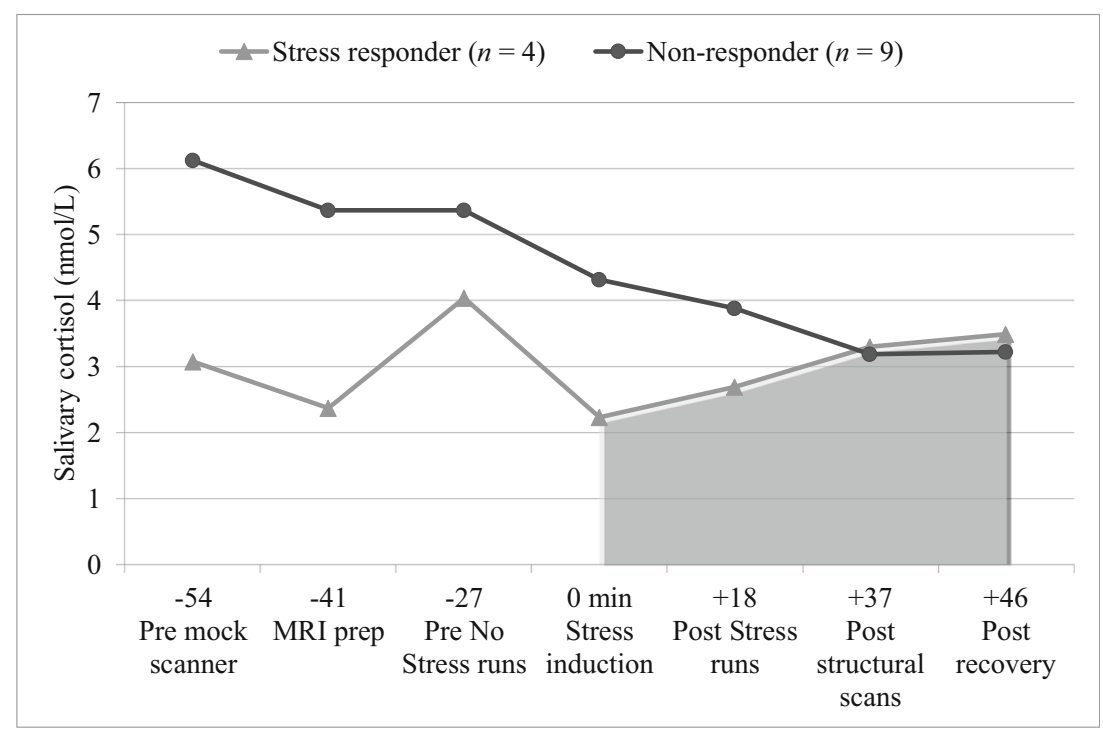


Table 1 The distribution of scores on personality tests in the total sample $(n=13)$ and stress responders $(n=4)$ and nonresponders $(n=9)$. Significant correlations with salivary cortisol (AUC with respect to increase) in response to stressor exposure are starred

\begin{tabular}{|c|c|c|c|}
\hline \multirow[b]{2}{*}{ Measurement tool } & \multirow{2}{*}{$\begin{array}{l}\text { Total sample } \\
M(S D)\end{array}$} & \multicolumn{2}{|c|}{ Cortisol subgroups } \\
\hline & & $\begin{array}{l}\text { Stress responder } \\
M(S D)\end{array}$ & $\begin{array}{l}\text { Non-responder } \\
M(S D)\end{array}$ \\
\hline \multicolumn{4}{|l|}{ MPQ-BF } \\
\hline Stress Reaction & $46.0(10.8)$ & $51.3(10.8)$ & $43.7(10.6)$ \\
\hline Social Closeness & $54.2(11.5)$ & $49.3(8.4)$ & $56.3(12.4)$ \\
\hline \multicolumn{4}{|l|}{ NEO-PI-R ${ }^{\mathrm{a}}$} \\
\hline Neuroticism & $52.9(12.9)$ & $63.3(5.7)$ & $48.3(12.6)$ \\
\hline Anxiety & $55.3(10.7)$ & $66.3(7.2)$ & $50.4(8.0)$ \\
\hline Angry Hostility & $52.2(11.7)$ & $60.8(6.3)$ & $48.4(11.7)$ \\
\hline Depression & $49.8(11.8)$ & $59.5(6.6)$ & $45.6(11.2)$ \\
\hline Self-Consciousness & $52.9(12.6)$ & $60.5(6.0)$ & $49.6(13.5)$ \\
\hline Impulsiveness & $51.9(12.9)$ & $55.3(9.2)$ & $50.4(14.5)$ \\
\hline Vulnerability & $51.4(11.1)$ & $55.8(11.4)$ & $49.4(11.1)$ \\
\hline Extraversion & $56.7(14.9)$ & $52.8(4.0)$ & $58.4(17.7)$ \\
\hline Warmth & $50.6(16.1)$ & $47.0(8.0)$ & $52.2(18.9)$ \\
\hline Gregariousness & $53.4(15.7)$ & $47.3(9.9)$ & $56.1(17.5)$ \\
\hline Assertiveness & $54.2(12.8)$ & $46.8(11.8)$ & $57.6(12.3)$ \\
\hline Activity* & $54.1(10.1)$ & $48.3(9.4)$ & $56.7(9.8)$ \\
\hline Excitement-Seeking & $57.8(10.2)$ & $63.5(6.6)$ & $55.2(10.8)$ \\
\hline Positive Emotions & $56.0(11.4)$ & $54.8(11.1)$ & $56.6(12.1)$ \\
\hline Openness & $58.1(9.8)$ & $62.8(10.5)$ & $56.0(9.3)$ \\
\hline Fantasy & 60.3 (11.6) & $68.0(10.5)$ & $56.9(10.9)$ \\
\hline Aesthetics & $54.5(13.2)$ & $59.0(11.8)$ & $52.6(14.0)$ \\
\hline Feelings & $56.7(10.1)$ & $58.8(9.4)$ & $55.8(10.8)$ \\
\hline Actions & $49.9(10.6)$ & $53.5(12.4)$ & $48.3(10.1)$ \\
\hline Ideas & $55.7(7.9)$ & $55.5(7.9)$ & $55.8(8.4)$ \\
\hline Values & $54.1(10.1)$ & $53.5(12.9)$ & $54.3(9.5)$ \\
\hline Agreeableness & $47.0(9.4)$ & $46.8(3.9)$ & $47.1(11.2)$ \\
\hline Trust & $47.2(12.2)$ & $38.8(8.3)$ & $50.9(12.1)$ \\
\hline Straightforwardedness & $47.6(8.4)$ & $48.8(6.2)$ & $47.1(9.5)$ \\
\hline Altruism & $51.4(11.6)$ & $54.5(8.7)$ & $50.0(12.9)$ \\
\hline Compliance & $45.9(8.9)$ & $42.5(6.0)$ & $47.4(9.9)$ \\
\hline Modesty & $49.4(11.4)$ & $58.0(15.5)$ & $45.6(7.1)$ \\
\hline Tender-Mindedness & $47.8(7.5)$ & $47.0(2.4)$ & $48.2(9.1)$ \\
\hline Conscientiousness & $46.6(17.0)$ & $33.8(9.8)$ & $52.3(16.7)$ \\
\hline Competence & $48.6(14.0)$ & $42.0(7.3)$ & $51.6(15.6)$ \\
\hline Order & $46.7(12.8)$ & $38.0(11.7)$ & $50.6(11.9)$ \\
\hline Dutifulness & $48.0(12.7)$ & $41.5(5.0)$ & $50.9(14.2)$ \\
\hline Achievement Striving* & $49.5(15.8)$ & $37.5(11.8)$ & $54.8(14.8)$ \\
\hline Self-Discipline & $43.4(16.1)$ & $28.3(7.9)$ & $50.1(14.1)$ \\
\hline Deliberation & $49.2(15.0)$ & $42.8(11.6)$ & $52.1(16.0)$ \\
\hline \multicolumn{4}{|l|}{ BIS/BAS } \\
\hline BIS subscore ${ }^{b}$ & $20.5(3.2)$ & $18.8(4.6)$ & $21.2(2.2)$ \\
\hline \multicolumn{4}{|l|}{ Rosenberg $^{\mathrm{c}}$} \\
\hline Total score & $22.2(6.0)$ & $17.8(6.3)$ & $24.1(4.9)$ \\
\hline
\end{tabular}

${ }^{\mathrm{a}} \mathrm{MPQ}-\mathrm{BF}$ and NEO-PI-R scores are $T$ scores.

${ }^{\mathrm{b}}$ BIS ranges from 7 to $28 .{ }^{\mathrm{c}}$ Rosenberg scores range from 0 to 30 with $<15$ indicating low self-esteem *Negative correlations with salivary cortisol AUC with respect to increase; significant at the .05 level 
Fig. 3 Ratings of perceived fear based on the Positive and Negative Affect ScalesExpanded Form (PANAS-X) by condition. Stress ratings were significantly greater than ratings for either No Stress or recovery, $p<.001$

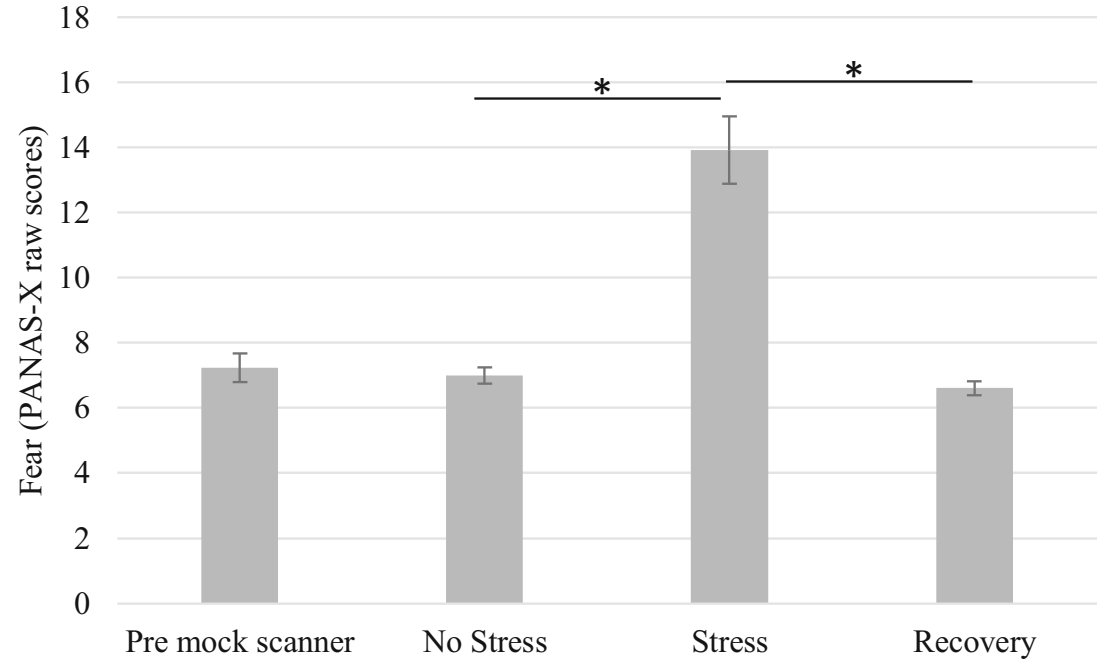

\section{Vocal function}

The mean Voice Handicap Index scores were $17.0(S D=16.4)$ in the total sample, $26.8(S D=22.2)$ for stress responders, and
12.7 ( $S D=12.3$ ) for non-responders. Regarding voicing efficiency analyses, data from one participant were missing due to experimenter error and subglottal pressure data from four participants did not meet quality criteria. For the remaining twelve

\section{a. Voice No Stress}

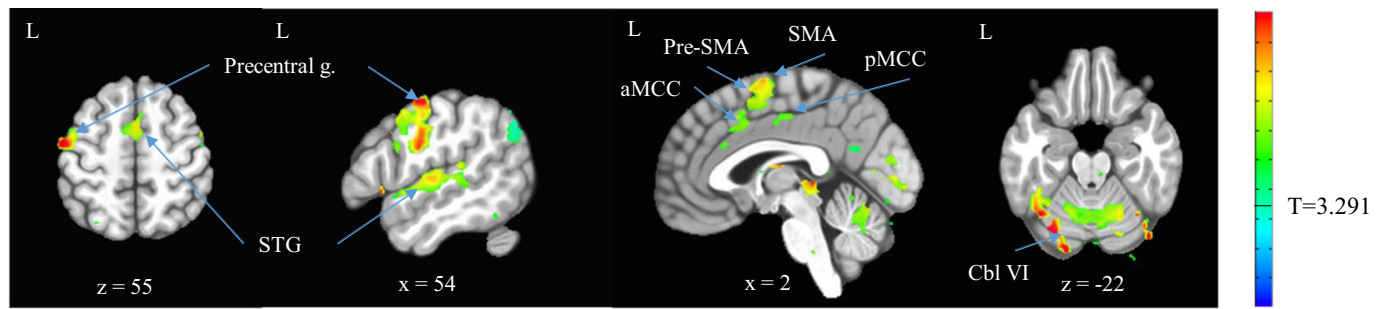

b. Voice Stress

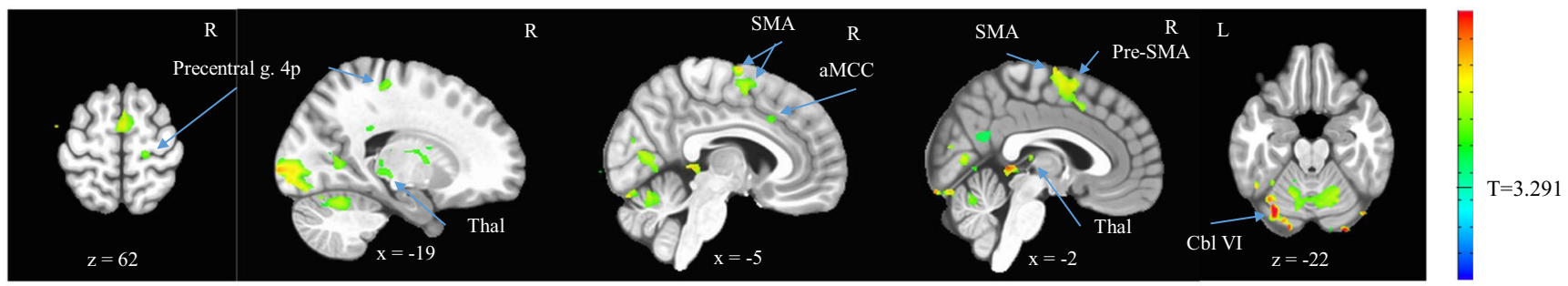

c. Voice Stress-Voice No Stress

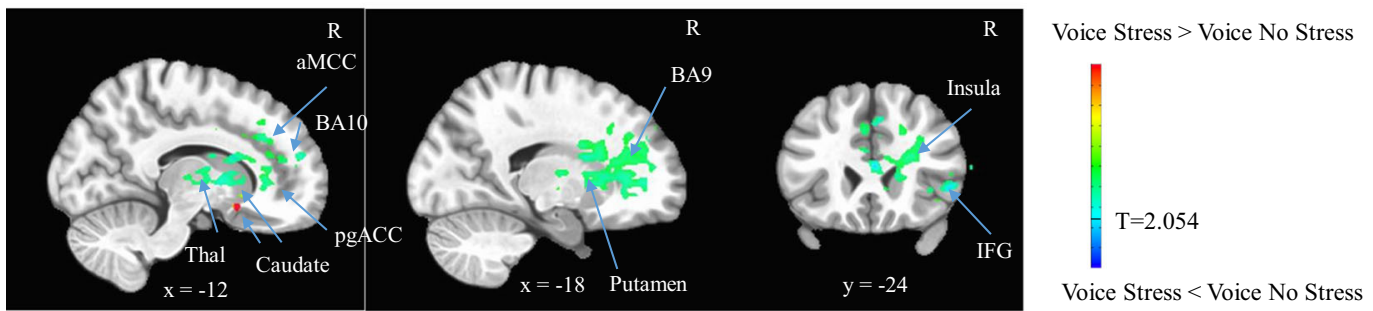

Fig. 4 BOLD activation maps for the conditions (a). Voice No Stress (VoiceNS) minus rest, (b). Voice Stress (VoiceS) minus rest, and the contrast (c). VoiceS minus VoiceNS over rest. The $t$ statistical parametric maps were thresholded at $p=.001$ (VoiceNS and VoiceS) and $p=.04$ (contrast VoiceNS vs. VoiceS) to achieve a family-wise error corrected $p<.05$. The contrast showed stressor-induced activations (red) and deactivations (green) in the caudate and deactivations (green/blue) in the right perigenual anterior cingulate cortex (pgACC), anterior middle cingulate cortex (aMCC), inferior frontal gyrus (IFG), BA 9, BA 10, insula, putamen, and thalamus (Thal). Precentral g. $=$ precentral gyrus, $\mathrm{STG}=$ superior temporal gyrus, $\mathrm{SMA}=$ supplementary motor area, $\mathrm{Cbl} \mathrm{VI}=$ cerebellum lobule VI 
Table 2 Activated brain regions for the Voice No Stress minus rest and Voice Stress minus rest conditions in the total sample $(n=13, t=3.291$, uncorrected $p=.001$, family-wise error corrected $p<.05$, minimum cluster size 7 voxels)

\begin{tabular}{|c|c|c|c|c|c|}
\hline \multirow[t]{2}{*}{ Anatomical Region } & \multicolumn{3}{|c|}{ Peak MNI Coordinates } & \multirow[t]{2}{*}{ Cluster Size in Voxels } & \multirow[t]{2}{*}{$p$ value, corrected } \\
\hline & $\mathrm{x}$ & $\mathrm{y}$ & $\mathrm{z}$ & & \\
\hline \multicolumn{6}{|l|}{ Voice No Stress } \\
\hline L Precentral g. & -54 & -9 & 55 & 1199 & $<.001$ \\
\hline L Cerebellum (VI) & -33 & -72 & -22 & 865 & $<.001$ \\
\hline R Superior temporal g. & 68 & -16 & 13 & 423 & $<.001$ \\
\hline L Inferior parietal lobule & -51 & -72 & 44 & 82 & $<.001$ \\
\hline L Superior parietal lobule & -26 & -69 & 48 & 18 & $<.01$ \\
\hline R Brainstem & 5 & -30 & -43 & 15 & $<.01$ \\
\hline L Precuneus & -2 & -58 & 23 & 14 & $<.01$ \\
\hline L Fusiform g. & -26 & -37 & -15 & 11 & $<.01$ \\
\hline L Caudate n. & -16 & 19 & 20 & 11 & $<.01$ \\
\hline L Middle occipital g. & -26 & -72 & 30 & 9 & $<.02$ \\
\hline R Cuneus & 5 & -100 & 27 & 9 & $<.02$ \\
\hline L Middle cingulate g. & -2 & -16 & 44 & 8 & $<.03$ \\
\hline R Lingual g. & 30 & -48 & -8 & 7 & $<.05$ \\
\hline L Cuneus & -16 & -62 & 20 & 7 & $<.05$ \\
\hline L Medial frontal $g$. & -23 & 40 & 16 & 7 & $<.05$ \\
\hline \multicolumn{6}{|l|}{ Voice Stress } \\
\hline L STG & -68 & -9 & 6 & 657 & $<.001$ \\
\hline R STG & 68 & -16 & 13 & 519 & $<.001$ \\
\hline L Cerebellum (VI) & -33 & -72 & -22 & 290 & $<.001$ \\
\hline R Inf. occipital g. & 26 & -90 & -22 & 273 & $<.001$ \\
\hline R SMA & 2 & -6 & 72 & 97 & $<.001$ \\
\hline L Angular g. & -54 & -69 & 37 & 28 & $<.001$ \\
\hline L Middle occipital g. & -30 & -72 & 27 & 18 & $<.01$ \\
\hline L Inf. temporal g. & -47 & -51 & -26 & 17 & $<.01$ \\
\hline L Angular g. & -40 & -62 & 30 & 14 & $<.01$ \\
\hline Cerebellar vermis & 2 & -41 & 2 & 11 & $<.01$ \\
\hline L Precuneus & -2 & -62 & 27 & 11 & $<.01$ \\
\hline L Inf. parietal lobule & -30 & -51 & 44 & 11 & $<.01$ \\
\hline R Precentral g. (4p) & 19 & -27 & 62 & 10 & $<.02$ \\
\hline $\mathrm{R} \mathrm{MCC}$ & 5 & 19 & 34 & 9 & $<.02$ \\
\hline R Cerebellum (VIIa) & 47 & -72 & -29 & 7 & $<.05$ \\
\hline R Lingual g. & 2 & -93 & -15 & 7 & $<.05$ \\
\hline L ParaHippocampal g. & -26 & -44 & -8 & 7 & $<.05$ \\
\hline R Caudate n. & 26 & -41 & 20 & 7 & $<.05$ \\
\hline
\end{tabular}

$M C C$ middle cingulate cortex, $M T G$ middle temporal gyrus, SMA supplementary motor area, STG superior temporal gyrus participants, airflow during voicing was $0.14 \mathrm{~L} / \mathrm{s}(S D=0.05)$. Subglottal pressure $\left(\mathrm{P}_{\text {sub }}\right)$ and laryngeal airway resistance $\left(\mathrm{R}_{\text {law }}\right)$ for eight participants with complete datasets were 7.64 $\mathrm{cm} \mathrm{H}_{2} \mathrm{O}(S D=1.26)$ and $71.31 \mathrm{~cm} \mathrm{H}_{2} \mathrm{O} /(\mathrm{L} / \mathrm{s})(S D=49.17)$, respectively. Thus, voicing efficiency data were within the normal range (Zraick et al. 2012). Stress responders had lower airflow during voicing than non-responders: stress responders $\mathrm{P}_{\text {sub }} 7.10 \mathrm{~cm} \mathrm{H}_{2} \mathrm{O}(S D=1.74)(n=3)$, airflow during voicing $0.10 \mathrm{~L} / \mathrm{s}(S D=0.04)$, and $\mathrm{R}_{\text {law }} 91.45$ $\mathrm{cm} \mathrm{H}_{2} \mathrm{O} /(\mathrm{L} / \mathrm{s})(S D=79.87)(n=3)$; non-responders $\mathrm{P}_{\text {sub }} 7.97$ $\mathrm{cm} \mathrm{H}_{2} \mathrm{O}(S D=0.95)(n=5)$, airflow during voicing $0.17 \mathrm{~L} / \mathrm{s}$ $(S D=0.05)$, and $\mathrm{R}_{\text {law }} 59.23 \mathrm{~cm} \mathrm{H}_{2} \mathrm{O} /(\mathrm{L} / \mathrm{s})(S D=23.56)(n=$ 5 ). Further, intensity and $f_{\mathrm{o}}$ differed between stress responders [SPL during voicing $76.4 \mathrm{~dB}(S D=4.1)$, mean $f_{\mathrm{o}} 213.1 \mathrm{~Hz}$ $(S D=40.23)]$ and non-responders [SPL during voicing $78.1 \mathrm{~dB}(S D=3.6)$, mean $\left.f_{\mathrm{o}} 190.8 \mathrm{~Hz}(S D=25.4)\right]$. Correlations with cortisol reactivity $\left(\mathrm{AUC}_{\mathrm{I}}\right)$ values were non-significant. 
Table 3 Activated brain regions for the contrasts Voice Stress minus Voice No Stress over rest and Whisper Stress minus Whisper No Stress over rest in the total sample $(n=13, t=2.054$, uncorrected $p=.04$, family-wise error corrected $p<.05$, minimum cluster size 469 voxels and 623 voxels, respectively)

\begin{tabular}{|c|c|c|c|c|c|}
\hline \multirow[t]{2}{*}{ Anatomical region } & \multicolumn{3}{|c|}{ Peak MNI Coordinates } & \multirow[t]{2}{*}{ Cluster Size in Voxels } & \multirow[t]{2}{*}{$p$ value, corrected } \\
\hline & $\mathrm{x}$ & $\mathrm{y}$ & $\mathrm{z}$ & & \\
\hline \multicolumn{6}{|c|}{ Voice Stress > Voice No Stress } \\
\hline R Caudate $\mathrm{n}$. & 12 & 15 & -12 & 472 & $<.05$ \\
\hline \multicolumn{6}{|c|}{ Whisper Stress $<$ Whisper No Stress } \\
\hline L ParaHippocampal g. & -2 & -34 & 2 & 727 & $<.05$ \\
\hline
\end{tabular}

\section{Neuroimaging data}

\section{Whole brain analyses}

\section{Brain activation in the total sample}

Figure 4 shows the brain areas activated in the total sample for the VoiceNS and VoiceS conditions separately, as well as the contrast of the two conditions after removal of baseline activation (rest). The contrast showed stressor-induced activations and deactivations in the caudate and deactivations in the right perigenual ACC (pgACC), anterior MCC (aMCC), inferior frontal gyrus (IFG, pars triangularis), BA 9, BA 10, insula, putamen, and thalamus. Tables 2 and 3 present an exhaustive list of all activated regions.

Figure 5 shows BOLD activation maps for the contrasts of WhisperS minus WhisperNS over rest as well as WhisperS minus VoiceS over rest. For the Whisper conditions, stressor exposure resulted in deactivation of the parahippocampal gyrus (Table 3). Contrasting Whisper and Voice conditions during Stress, with the aim of revealing laryngeal-related activations, showed greater activations in the precentral gyrus (area 6) and less activations in the STG for the Whisper than the Voice condition during Stress (Table 4). Activity in area 6 was in close proximity to the dorsolateral LMC described by Brown et al. (2008, 2009). In addition, Table 4 lists brain regions activated for the contrast of Whisper and Voice during the No Stress condition. For this contrast, brain activations were greater in the left MFG and right precentral gyrus for the Whisper than the Voice condition.

\section{ROI analyses}

Figure 6 shows mean percent BOLD signal changes in ROIs for the VoiceNS and VoiceS conditions. BOLD signals decreased in the corticolimbic areas MFG, ACC, amygdala, HC, thalamus, and PAG. In the primary vocalization areas, $\mathrm{L}$ area $4 \mathrm{p}$ motor activity increased and L SMA and L cerebellum lobule VI activity decreased. In the secondary vocalization areas, responses in the MCC and insula remained stable overall but activity in the putamen and thalamus decreased. In summary, stressor exposure was characterized by increases in sensorimotor activity and decreases in SMA, cerebellum, and limbic activity.

Correlations of BOLD activations in ROIs with salivary cortisol, personality, and aerodynamic vocal function Figure 7 shows all significant correlations at $p<.05$ and $\mathrm{L}$ area $4 \mathrm{p}$ correlations between salivary cortisol responses $\left(\mathrm{AUC}_{\mathrm{I}}\right)$ and mean percent BOLD signal change in ROIs during the VoiceNS and VoiceS conditions. For VoiceNS, there was a strong negative correlation for cortisol reactivity with signal changes in the $\mathrm{L}$ area $4 \mathrm{p}$ $(r=-.81, p=.001)$ and moderate positive correlations with the L SMA $(r=.56, p=.045)$. For VoiceS, a moderately strong correlation emerged for the L ACC $(r=.61, p=.027)$. Further, multiple moderate correlations were significant at $p<.1$ including, in order of significance, right thalamus $(r=.54, p=.058), \mathrm{L} 4 \mathrm{p}(r=-.51, p=.072), \mathrm{L} \operatorname{MCC}(r=.50$, $p=.082)$, and L HC $(r=.50, p=.086)$.

The above mentioned ROIs were significantly correlated with selected personality traits and aerodynamic measures. For the active control condition, Voice No Stress, L area $4 p$ was strongly correlated with Agreeableness-Modesty $(r=$ $-.71, p=.006)$ and moderately correlated with airflow $(r=.68, p=.015)$, Neuroticism-Depression $(r=-.65$, $p=.016)$, self-esteem $(r=.63, p=.020)$, ExtraversionActivity $(r=.60, p=.029)$, Conscientiousness-Achievement Striving $(r=.57, p=.042)$, and Extraversion $(r=.50$, $p=.079)$. In addition, airflow was also moderately correlated with PAG activity $(r=-.59, p=.046)$ but with no further brain region. Further, L SMA was also moderately correlated with Extraversion-Activity $(r=-.51, p=.073)$ as well as Agreeableness-Altruism $(r=.51, p=.072)$ and Agreeableness-Straightforwardedness $(r=.48, p=.100)$.

For the active task condition, Voice Stress, L area $4 \mathrm{p}$ was moderately correlated with BIS scores $(r=.67, p=.013)$ and again Conscientiousness-Achievement Striving $(r=.59$, $p=.035)$. Further, the L ACC was moderately correlated with $\mathrm{P}_{\text {sub }}(r=-.67, p=.071)$, the $\mathrm{R}$ thalamus was moderately correlated with Extraversion-Gregariousness $(r=.49, p=.088)$, and the L hippocampus was moderately correlated with OpennessIdeas $(r=.69, p=.009), \mathrm{R}_{\text {law }}(r=-.67, p=.071)$, Agreeableness-Straightforwardedness $(r=.64, p=.018)$, 


\section{a. Whisper Stress-Whisper No Stress}

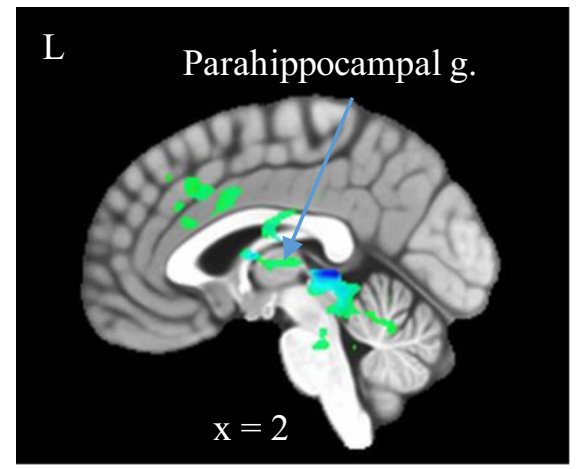

Whisper Stress $>$ Whisper No Stress

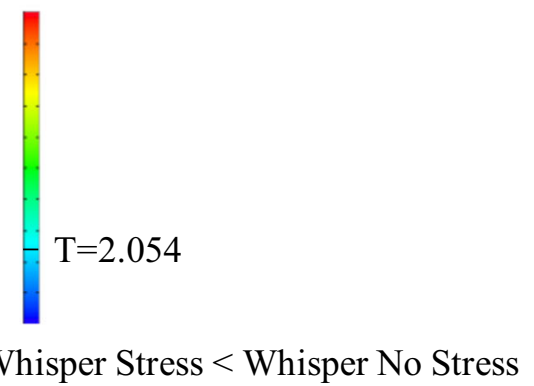

b. Whisper Stress-Voice Stress
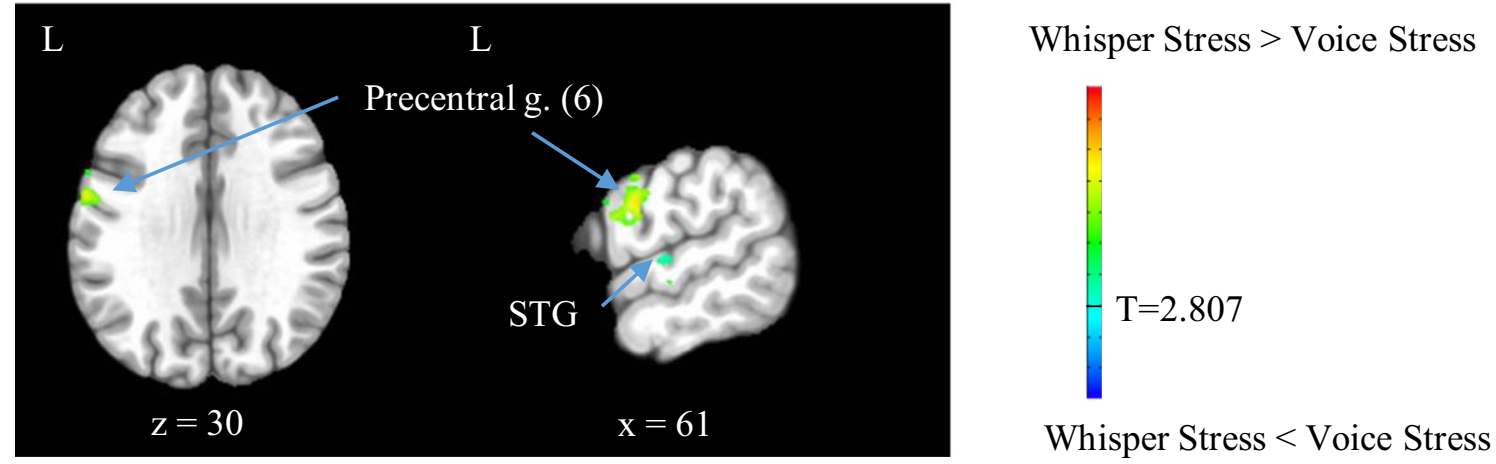

Fig. 5 BOLD activation maps for the contrasts (a). Whisper Stress (WhisperS) minus Whisper No Stress (WhisperNS) over rest and (b). WhisperS minus VoiceS over rest. The $t$ statistical parametric maps were thresholded at $p=.04$ (WhisperS vs. WhisperNS) and $p=.005$ (WhisperS vs. VoiceS) to achieve a family-wise error corrected $p<.05$. For the

Agreeableness $(r=.53, p=.060)$, and AgreeablenessCompliance $(r=.49, p=.092)$. The L MCC did not have significant correlations.

To summarize, the lower a participant's $L$ area $4 p$ activity was, the lower the person's Extraversion-Activity, ConscientiousnessAchievement Striving, self-esteem, and behavioral inhibition scores and the higher the person's Neuroticism-Depression and Agreeableness-Modesty scores. Lower Extraversion-Activity also correlated with greater $\mathrm{AUC}_{\mathrm{I}}$ values and L SMA activations. With regard to aerodynamic measures, lower $\mathrm{L}$ area $4 \mathrm{p}$ activity correlated with lower airflow during No Stress. During Stress, lower $\mathrm{P}_{\text {sub }}$ and greater $\mathrm{R}_{\text {law }}$ correlated with greater $\mathrm{L}$ ACC and lower $\mathrm{L} \mathrm{HC}$ activity, respectively.

\section{Discussion}

\section{Effects of stress on brain activations in the total sample}

The first aim of this study was to determine the effect of social-evaluative stress on the central neural control of
Whisper condition, stressor exposure resulted in deactivation of the parahippocampal gyrus (parahippocampal g.). Contrasting Whisper and Voice during Stress showed increased activations in the precentral gyrus (precentral g. area 6) and decreased activations in the superior temporal gyrus (STG)

phonation underlying speech production. First, brain activations during the VoiceNS condition were largely consistent with the known speech production network underlying sentence reading (Fuertinger et al. 2015; Simonyan and Fuertinger 2015) apart from activations for visual processing, memory, and reading. The activated clusters encompassed the LMC (area 4p), IFG, SMA, STG, cingulate cortex, putamen, thalamus, and cerebellum (lobule VI). The stress induction was successful as shown by a significant increase in perceived fear during speech anticipation. The task contrast (VoiceSVoiceNS) revealed that stressor exposure was characterized by a peak activation in the right caudate, as predicted, with concomitant deactivations in the bilateral pgACC and aMCC, and right IFG, BA 9, BA 10, insula, putamen, and thalamus. Thus, a significant effect on primary or premotor cortices was not detected.

The key impact of stressor exposure on the vocal control system occurred in areas considered secondary to phonation control, specifically deactivations in the ACC and MCC, insula, putamen, and thalamus. Except for the direction of the activation changes, our findings parallel those from an early PET study by Paus et al. (1993) whose experimental design required a simple one-word response, which was or 
Table 4 Activated brain regions for the contrasts Whisper No Stress minus Voice No Stress over rest and Whisper Stress minus Voice Stress over rest in the total sample $(n=13, t=2.807$, uncorrected $p=.005$, family-wise error corrected $p<.05$, minimum cluster size 17 voxels and 20 voxels, respectively)

\begin{tabular}{|c|c|c|c|c|c|}
\hline \multirow[t]{2}{*}{ Anatomical region } & \multicolumn{3}{|c|}{ Peak MNI Coordinates } & \multirow[t]{2}{*}{ Cluster Size in Voxels } & \multirow[t]{2}{*}{$p$ value, corrected } \\
\hline & $\mathrm{x}$ & $\mathrm{y}$ & $\mathrm{z}$ & & \\
\hline \multicolumn{6}{|c|}{ Whisper No Stress > Voice No Stress } \\
\hline L Middle frontal g. & -61 & 1 & 41 & 99 & $<.001$ \\
\hline R Precentral g. & 68 & -16 & 41 & 77 & $<.001$ \\
\hline \multicolumn{6}{|c|}{ Whisper Stress < Voice Stress } \\
\hline L Superior temporal g. & -68 & -13 & 2 & 35 & $<.02$ \\
\hline \multicolumn{6}{|c|}{ Whisper Stress $>$ Voice Stress } \\
\hline L Precentral g. (6) & -61 & -2 & 30 & 28 & $<.03$ \\
\hline
\end{tabular}

was not in conflict with an overlearned stimulus-response alternative. Their study yielded primarily activations in the left paracingulate gyrus (BA 32/8) as well as in the rostral ACC (BA 24), BA 10, and BA 9/45. Our predictions were partially based on a study by Lorberbaum et al. (2004) that compared the effects of anticipation of public speaking in healthy controls and individuals with generalized social phobia. Our results are in striking concordance with those in the social phobia group instead of the control group. The social phobia group showed less activation in the left aMCC, medial PFC (BA 8,32), and dIPFC (BA 9) than the control group during stressor exposure. The authors concluded that limbic hyperactivation with cortical hypoactivation led to their participants not being able to "think clearly" (p. 2703). A reason for the activation directions of our results may be that we pre-selected our sample to include a comparable number of individuals who scored above and below the norm on stress reactivity instead of a random distribution.

The ACC and MCC are key regions because of potentially degrading effects on voice production. The ACC is involved in voice initiation and emotions underlying prosody (Simonyan and Horwitz 2011). However, simple tasks such as syllable productions do not generally evoke these activations whereas complex tasks such as reading or speaking do engage the prefrontal cortex and involve prosody and emotional vocalizations mediated by the cingulate cortex (Fuertinger et al. 2015; Simonyan and Fuertinger 2015; Simonyan and Horwitz 2011; Simonyan et al. 2009). The ACC and MCC transform intentions into actions via the neuromotor system, integrate cognitions via the lateral PFC, and modulate fear and arousal via the thalamus, amygdala, and PAG (Etkin et al. 2011; Paus 2001; Vogt 2016). The aMCC may influence vocal behavior through its role in conflict monitoring and (willed) action selection as the dorsal aMCC is pivotal to action anticipation, response initiation, and monitoring of ongoing action outcomes (Etkin et al. 2011; Vogt 2016).

Thus, the relative drop in activation in the aMCC may be linked to diminished guidance for motor choices and consequently disrupted implementation of voice as the aMCC (a) co-activates with the lateral PFC and MFG (Paus 2001; Paus et al. 1993), (b) connects with the SMA (Etkin et al.), and (c) maintains reciprocal connectivity with the LMC (Simonyan and Horwitz 2011). The LMC also has reciprocal connectivity with the dlPFC as well as with the insula and thalamus (Simonyan and Horwitz 2011). While the anterior insula appears to be involved in both articulation and phonation, its exact vocal motor role is still debated (Brown et al. 2009). The ventral tier nuclei of the thalamus has a well-established role in vocal sensorimotor coordination of learned voice production including the integration of information from the basal ganglia and cerebellum (Simonyan and Horwitz 2011). The putamen, which is also involved in learned voice production, often co-activates with the thalamus but only receives input from the LMC and does not feed back to the LMC (Simonyan and Horwitz 2011).

Most relevant in the context of stress, subcortical structures modulate the cognitive motor input received by the ACC and MCC in challenging situations, leading either to the facilitation or suppression of motor responses (Paus et al. 1993). The ACC and MCC directly connect with limbic structures including the amygdala, PAG, and HY (Etkin et al.; Vogt 2016). The pgACC, which is involved in emotion and autonomic regulation (Vogt 2016), can exert top-down control to inhibit negative emotional processing in the amygdala (Etkin et al. 2011). This regulatory role might have been diminished considering a stressor-induced drop in pgACC activity and heightened scores of perceived fear. Emotion regulation must be relevant for voice production because the amygdala may modulate LMC activity via the SMA, in extremes leading to signs of motor conversion (Boeckle et al. 2016; Simonyan and Fuertinger 2015). Overall, given the deactivation in the thalamus, connected subcortical areas, and the cerebellum, motor programming and motor execution was likely altered, possibly degraded during stress. However, across participants, the final sensorimotor output in primary vocalization areas (i.e., LMC, premotor) was not significantly different between Voice No Stress and Stress conditions. 
$\because$ VoiceNS $\backsim$ VoiceS

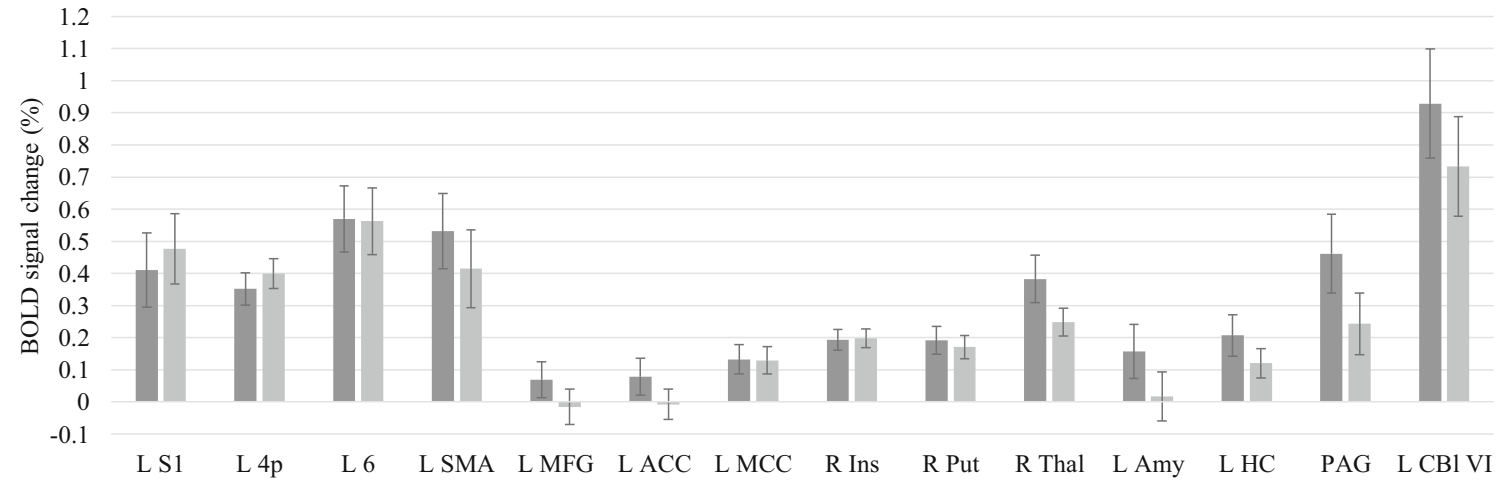

Fig. 6 Brain responses in mean percent BOLD signal change in regions of interest (ROI) for voice productions during the No Stress and Stress conditions. The ROIs represent regions involved in voice and speech production and stress responses with consideration of lateralization. $\mathrm{L} / \mathrm{R}=$ left/right, $\mathrm{S} 1=$ postcentral gyrus, $4 \mathrm{p}=$ posterior part of area $4,6=$

\section{Brain activations for the contrast of voiced versus whispered speech}

The contrast of voiced and whispered productions was included to better localize stressor-induced brain changes for vocalization. During the Stress condition, there was less activation in the left STG and more activation in the left precentral gyrus
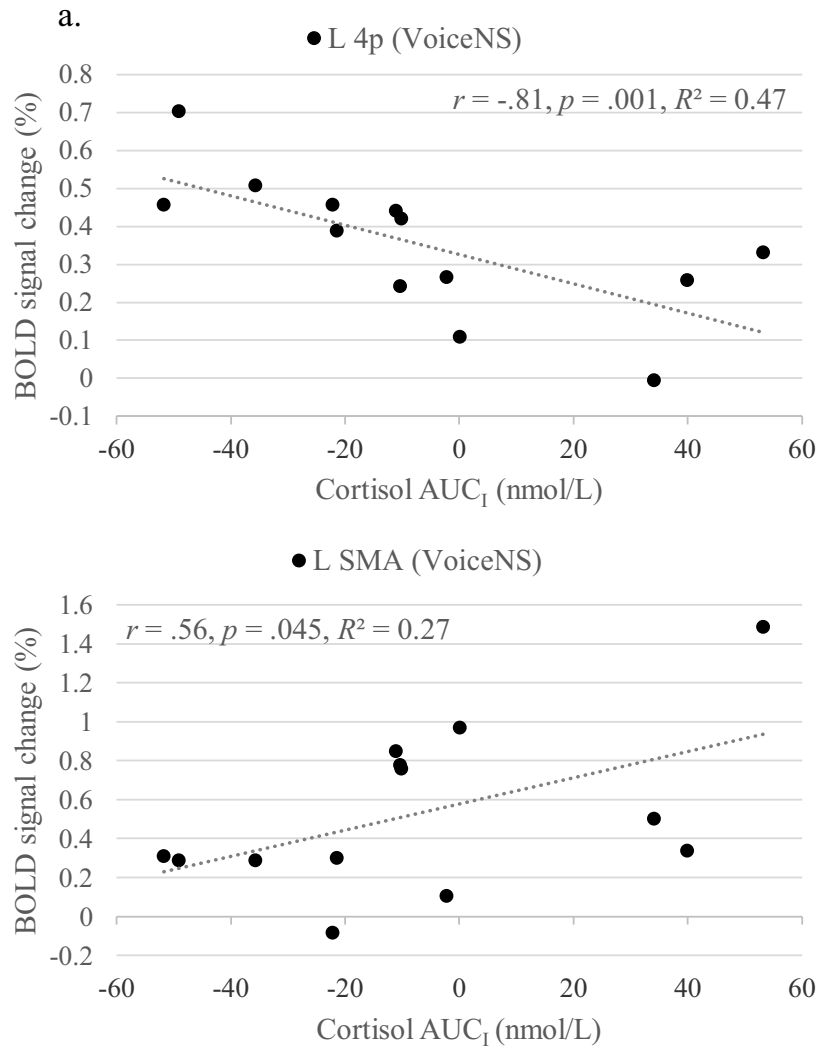

Fig. 7 Correlations between the cortisol response to the stressor (area under the curve with respect to increase $\left[\mathrm{AUC}_{\mathrm{I}}\right.$ in $\left.\mathrm{nmol} / \mathrm{L}\right]$ ) and mean percent BOLD signal changes during the conditions (a). Voice No Stress area $6, \mathrm{SMA}=$ supplementary motor area, $\mathrm{MFG}=$ middle frontal gyrus, $\mathrm{ACC}=$ anterior cingulate cortex, $\mathrm{MCC}=$ middle cingulate cortex, Ins $=$ insula, Put $=$ putamen, Thal $=$ thalamus, Amy $=$ amygdala, $\mathrm{HC}=$ hippocampus, $\mathrm{PAG}=$ periaqueductal gray, and $\mathrm{Cbl}(\mathrm{VI})=$ Lobule VI of the cerebellum

(area 6) during the Whisper than Voice condition. The activation difference included the left dorsolateral LMC in area 6, which is an area that can be recruited for additional indirect control of laryngeal motoneurons in addition to the LMC in area $4 p$ (Simonyan 2014). The greater activation in area 6 for whispered productions during the Stress condition may reflect heightened motor cortical drive to inhibit full laryngeal

b.

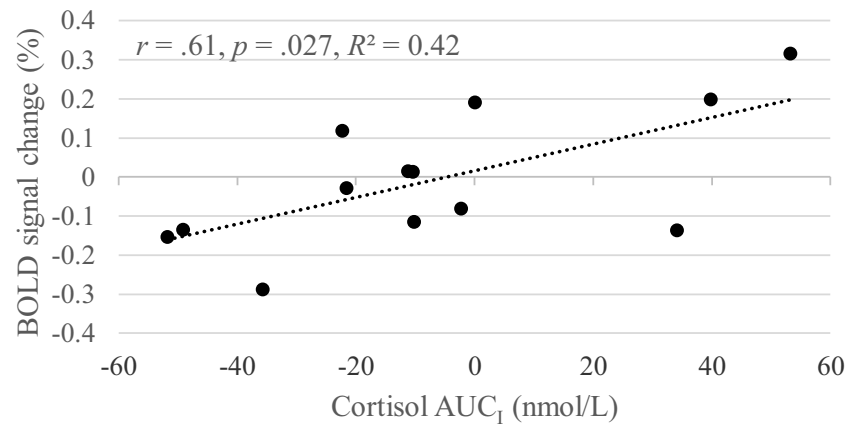

- L $4 p$ (VoiceS)

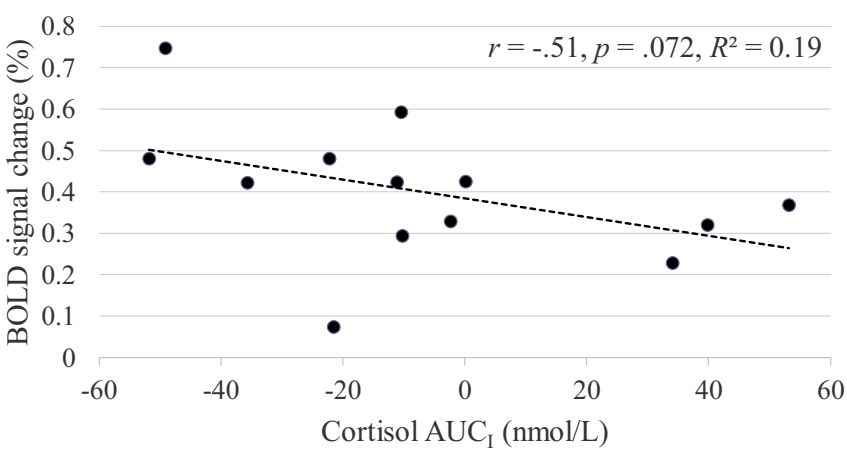

(VoiceNS) and (b). Voice Stress (VoiceS) in the left (L) area 4p, L supplementary motor area (SMA), and L anterior cingulate cortex (ACC) 
engagement and posture the glottis in a partially opened state. Increased activation in this region during the Whisper condition may also reflect augmented drive associated with the emotional and cognitive consequences of the stressor condition.

\section{Correlations of BOLD activations in ROls with salivary cortisol, personality, and aerodynamic vocal function}

The second aim of this study was to determine the neural signature, personality profile, and aerodynamic vocal function in relation to salivary cortisol responses. Hypotheses were partially confirmed.

The hallmark of the Stress condition was a moderate positive correlation of cortisol reactivity with left ACC activation (to a lesser degree L MCC activation). As discussed in the previous section, the ACC and MCC are involved in conflict and action monitoring and heightened activity in stress responders may be linked with processing of emotions and cognitions related to the stressor with consequences for motor activity. The ACC and MCC connect with the SMA via the dIPFC. The (pre)-SMA modulates behavior, and by extension vocal behavior. The pre-SMA, a cognitive-motor area, may energize or inhibit behavior (Wager et al. 2009) while the SMA proper initiates motor behavior (Nachev et al. 2008; Picard and Strick 2001). Only the SMA proper connects directly with the primary motor cortex (Etkin et al. 2011). The pre-SMA is capable of response inhibition or "braking" through "hyperdirect" connections with the subthalamic nucleus and belongs to a motor inhibition network encompassing the right inferior frontal cortex, pre-SMA, globus pallidus, and subthalamic nucleus (Aron et al. 2014). Relatedly, the SMA belongs to a motor conversion disorder network composed of the dIPFC, medial PFC, aMCC, superior frontal gyrus, insula, and amygdala where shifts in prefrontal and cingulate functioning can result in inhibition of motor behavior mediated by a dysfunctional SMA-amygdala connection (Boeckle et al. 2016).

The effect of stressor exposure on LMC activity became clearer once correlations of BOLD signal changes with cortisol data were investigated in addition to comparisons by condition. Cortisol reactivity had a strong negative correlation with $\mathrm{L}$ area $4 \mathrm{p}$ during Voice No Stress, which decreased to a moderate negative correlation during Voice Stress leading to a stressor-induced increase in $\mathrm{L}$ area $4 p$ activity shown in Fig. 6. That is, greater cortisol reactivity was generally linked to lower $L$ area $4 p$ activity. Left area $4 p$ is a critical participant in the initiation and execution of motor commands for speech and the modulation of movement-related attention (Fuertinger et al. 2015). Acute stress did appear to mobilize motor areas for speech production including the LMC in those who were more stress reactive perhaps increasing effort for the initiation and execution of motor commands for speech despite a conflicting communicative situation (wanting to avoid public speaking).

With regard to personality, a key finding was that data converged on a relationship of cortisol reactivity and laryngeal motor activity with extraversion. Greater cortisol reactivity and generally lower $\mathrm{L}$ area $4 \mathrm{p}$ activity was linked with lower Extraversion-Activity scores across conditions. In addition, lower $\mathrm{L}$ area $4 \mathrm{p}$ activity correlated with lower airflow during voicing during the No Stress condition. Thus, the data align with the Trait Theory of Voice Disorders in that extraversion may play an important role in the relationship between personality and laryngeal behavior, reflecting a less "activated" behavioral state.

During the Stress condition, greater $L$ area $4 p$ activity was linked with greater Conscientiousness-Achievement Striving and greater behavioral inhibition scores, personality attributes that characterized individuals who were less and not more cortisol reactive. During the Stress condition, lower $\mathrm{P}_{\text {sub }}$ and greater $\mathrm{R}_{\text {law }}$ correlated with greater L ACC (no correlation with personality) and lower L HC activity (lower OpennessIdeas, Agreeableness-Straightforwardedness), respectively. Perhaps the stressor-induced mobilization of area $4 p$ was partially the result of increased or altered laryngeal airway resistance (subglottal pressure divided by airflow during voicing) to meet the speaking demands during stress. Such an interpretation is supported by a separate study with cortisol data that showed a drop in airflow during voicing with stressor exposure in stress responders but not non-responders, which could be related to a tendency for breathholding (Dietrich, unpublished results). Overall, the stressor-induced neural signature might reflect heightened effort to produce speech in light of insufficient cortical input to initiate speech for at least some participants.

\section{Limitations and future directions}

The study's focus was on feasibility testing of a stress reactivity protocol coupled with speech production as well as obtaining pilot data. Therefore, the findings are limited by the study's small sample size. Nonetheless, existing significant findings and trends that underscore differential neural signatures underlying brain responses to stress are promising. Stress responder and non-responder subgroups were small and also imbalanced with only four stress responders although we pre-screened individuals on trait stress reactivity. One reason might be that some participants experienced cortisol reactivity in response to practice in the mock scanner (Fig. 2), which in turn limited the strength of the $\mathrm{AUC}_{\mathrm{I}}$ formula because it takes into account cortisol levels pre-stressor to identify stress responders. Despite recovery time post practice, only one cortisol data point pre-stressor could be used as baseline, instead of two or three. A practice session on a separate day or longer recovery would be a solution. 
There is the potential that a task repetition effect affected the results for the Stress condition. However, the stressor cannot precede the baseline condition. In fact, the pattern of brain activations differed as a function of cortisol reactivity thus supporting the effect of the stress manipulation on the results. The Voice-Whisper contrast may not have adequately isolated laryngeal activity. Whispering also includes laryngeal activity, but is produced with limited vocal fold adduction and no vocal fold vibration. However, interindividual variability in whispering has been recognized and complicates the interpretation of results (Andreatta et al. 2010; Konnai et al. 2017). Further, voice behavioral data were not collected during the MRI session but the accuracy of the tasks was monitored. Future studies should strive to collect acoustic and vocal function data during the MRI session. Finally, functional connectivity analyses will be an important next step to study pathways interconnecting ROIs. BOLD fMRI is inherently limited as the nature of the BOLD signal change in terms of activation or inhibition cannot be distinguished. Coactivation of regions does not equal functional relationships between and among activated regions.

\section{Conclusion}

To the best of our knowledge, this is the first study investigating the central neural effects of a public speaking speech preparation stressor on the phonatory control for speech. The study showed that our fMRI stress reactivity and speech protocol was feasible and that pilot data were promising showing that (a) the key impact of stressor exposure on vocal control occurred in areas considered secondary to phonation control, specifically deactivations in the ACC, MCC, insula, putamen, and thalamus, and (b) there are individual differences in stressor-induced brain activations as a function of stress reactivity, as defined by salivary cortisol, with greater cortisol reactivity linked with lower laryngeal motor cortex activity and lower scores on aspects of extraversion. Our preliminary findings are significant, because they illustrate how perturbations of the speech production system may interfere with the voluntary control of phonation via potential limbic-motor interactions. The individual differences lend preliminary support to key aspects of the Trait Theory of Voice Disorders that the limbic system modulates sensorimotor function underlying vocal control differently in those who are more reactive to novelty and threat. However, a link to heightened peripheral laryngeal muscle tension must be studied further. Our data lay the groundwork for future hypothesis-driven research on the effects of stress on voice production in individuals with and without voice disorders such as muscle tension dysphonia. The current findings are critical to interpret disordered processes underlying the central control of voice and psychobiological measures (cortisol, fear, personality). Laryngeal function indices provide important confirmatory correlates to our imaging data.

Acknowledgments We thank David K. Powell and Lexie Lusk, Michelle Rosh, and Kayla Sergesketter for assisting with data collection, and Ashwini Joshi for an earlier version of the E-Prime script.

Funding This work was supported by the National Center for Research Resources and the National Center for Advancing Translational Sciences [grant number UL1TR001998]; Center for Clinical and Translational Science Pilot Research Program, University of Kentucky; and the Magnetic Resonance Imaging and Spectroscopy Center Pilot Research Program, University of Kentucky.

\section{Compliance with ethical standards}

Conflict of interest The authors declare that they have no conflict of interest.

Ethical approval All procedures performed in studies involving human participants were in accordance with the ethical standards of the University of Kentucky Institutional Review Board and with the 1964 Helsinki declaration and its later amendments or comparable ethical standards. The article does not contain any studies with animals performed by any of the authors.

Informed consent Informed consent was obtained from all individual participants included in the study.

Open Access This article is distributed under the terms of the Creative Commons Attribution 4.0 International License (http:// creativecommons.org/licenses/by/4.0/), which permits unrestricted use, distribution, and reproduction in any medium, provided you give appropriate credit to the original author(s) and the source, provide a link to the Creative Commons license, and indicate if changes were made.

\section{References}

Andreatta, R. D., Stemple, J. C., Joshi, A., \& Jiang, Y. (2010). Taskrelated differences in temporo-parietal cortical activation during human phonatory behaviors. Neuroscience Letters, 484(1), 51-55. https://doi.org/10.1016/j.neulet.2010.08.017.

Aron, A. R., Robbins, T. W., \& Poldrack, R. A. (2014). Inhibition and the right inferior frontal cortex: One decade on. Trends in Cognitive Sciences, 18(4), 177-185. https://doi.org/10.1016/j.tics.2013.12. 003.

Boeckle, M., Liegl, G., Jank, R., \& Pieh, C. (2016). Neural correlates of conversion disorder: Overview and meta-analysis of neuroimaging studies on motor conversion disorder. BMC Psychiatry, 16, 195. https://doi.org/10.1186/s12888-016-0890-x.

Brown, S., Ngan, E., \& Liotti, M. (2008). A larynx area in the human motor cortex. Cerebral Cortex, 18(4), 837-845. https://doi.org/10. 1093/cercor/bhm131.

Brown, S., Laird, A. R., Pfordresher, P. Q., Thelen, S. M., Turkeltaub, P., \& Liotti, M. (2009). The somatotopy of speech: Phonation and articulation in the human motor cortex. Brain and Cognition, 70(1), 31-41. https://doi.org/10.1016/j.bandc.2008.12.006.

Carver, C. S., \& White, T. L. (1994). Behavioral inhibition, behavioral activation, and affective responses to impending reward and 
punishment: The BIS/BAS scales. Journal of Personality and Social Psychology, 67(2), 319-333.

Costa, P. T., \& McCrae, R. R. (1992). Revised NEO Personality Inventory (NEO-PI-R) professional manual. Odessa, FL: Psychological Assessment Resources.

Cox, R. W. (1996). AFNI: Software for analysis and visualization of functional magnetic resonance neuroimages. Computers and Biomedical Research, 29(3), 162-173. S0010480996900142 [pii].

Cox, R. W. (2012). AFNI: What a long strange trip it's been. Neuroimage, 62(2), 743-747. https://doi.org/10.1016/j.neuroimage.2011.08.056.

Dedovic, K., D'Aguiar, C., \& Pruessner, J. C. (2009a). What stress does to your brain: A review of neuroimaging studies. Canadian Journal of Psychiatry, 54(1), 6-15.

Dedovic, K., Duchesne, A., Andrews, J., Engert, V., \& Pruessner, J. C. (2009b). The brain and the stress axis: The neural correlates of cortisol regulation in response to stress. Neuroimage, 47(3), 864 871. https://doi.org/10.1016/j.neuroimage.2009.05.074.

Dickerson, S. S., \& Kemeny, M. E. (2004). Acute stressors and cortisol responses: A theoretical integration and synthesis of laboratory research. Psychological Bulletin, 130(3), 355-391. https://doi.org/10. 1037/0033-2909.130.3.355.

Dietrich, M., \& Verdolini Abbott, K. (2008). A psychobiological framework for studying psychological stress and its relation to voice disorders. In K. Izdebski (Ed.), Emotions in the human voice (vol.3) (pp. 159-178). San Diego: Plural.

Dietrich, M., \& Verdolini Abbott, K. (2012). Vocal function in introverts and extraverts during a psychological stress reactivity protocol. Journal of Speech, Language, and Hearing Research, 55(3), 973987. https://doi.org/10.1044/1092-4388(2011/10-0344).

Dietrich, M., \& Verdolini Abbott, K. (2014). Psychobiological stress reactivity and personality in persons with high and low stressorinduced extralaryngeal reactivity. Journal of Speech, Language, and Hearing Research, 57(6), 2076-2089. https://doi.org/10.1044/ 2014 jslhr-s-12-0386.

Dietrich, M., Verdolini Abbott, K., Gartner-Schmidt, J., \& Rosen, C. A. (2008). The frequency of perceived stress, anxiety, and depression in patients with common pathologies affecting voice. Journal of Voice, 22(4), 472-488. https://doi.org/10.1016/j.jvoice.2006.08.007.

Dietrich, M., Andreatta, R. D., Jiang, Y., Joshi, A., \& Stemple, J. C. (2012). Preliminary findings on the relation between the personality trait of stress reaction and the central neural control of human vocalization. International Journal of Speech-Language Pathology, 14, 377-389. https://doi.org/10.3109/17549507.2012.688865.

Eickhoff, S. B., Stephan, K. E., Mohlberg, H., Grefkes, C., Fink, G. R., Amunts, K., \& Zilles, K. (2005). A new SPM toolbox for combining probabilistic cytoarchitectonic maps and functional imaging data. Neuroimage, 25(4), 1325-1335. https://doi.org/10.1016/j. neuroimage.2004.12.034.

Etkin, A., Egner, T., \& Kalisch, R. (2011). Emotional processing in anterior cingulate and medial prefrontal cortex. Trends in Cognitive Sciences, 15(2), 85-93. https://doi.org/10.1016/j.tics.2010.11.004.

Fuertinger, S., Horwitz, B., \& Simonyan, K. (2015). The functional connectome of speech control. PLoS Biology, 13(7), e1002209. https://doi.org/10.1371/journal.pbio.1002209.

Haslinger, B., Erhard, P., Dresel, C., Castrop, F., Roettinger, M., \& Ceballos-Baumann, A. O. (2005). "silent event-related" fMRI reveals reduced sensorimotor activation in laryngeal dystonia. Neurology, 65(10), 1562-1569.

Helou, L. B. (2014). Intrinsic laryngeal muscle response to a speech preparation stressor: Personality and autonomic predictors. (Ph.D.), University of Pittsburgh, Pittsburgh.

Hillman, E., Holmberg, E. B., Perkell, J. S., Walsh, M., \& Vaughan, C. (1989). Objective assessment of vocal hyperfunction: An experimental framework and initial results. Journal of Speech and Hearing Research, 32, 373-392.
Jacobson, B. H., Johnson, A., Grywalski, C., Silbergleit, A., Jacobson, G., Benninger, M. S., \& Newman, C. W. (1997). The Voice Handicap Index (VHI): Development and validation. American Journal of Speech-Language Pathology, 6(3), 66-70. 1058-0360/ 97/0603-006.

Jenkinson, M., Beckmann, C. F., Behrens, T. E. J., Woolrich, M. W., \& Smith, S. M. (2012). FSL. Neuroimage, 62(2), 782-790. https://doi. org/10.1016/j.neuroimage.2011.09.015.

Jürgens, U. (2002). Neural pathways underlying vocal control. Neuroscience and Biobehavioral Reviews, 26(2), 235-258.

Kempster, G. B., Gerratt, B. R., Verdolini Abbott, K., BarkmeierKraemer, J., \& Hillman, R. E. (2009). Consensus auditoryperceptual evaluation of voice: Development of a standardized clinical protocol. American Journal of Speech-Language Pathology, 18(2), 124-132. 1058-0360/09/1802-012.

Kirschbaum, C., Pirke, K.-M., \& Hellhammer, D. (1993). The 'Trier Social Stress Test' - a tool for investigating psychobiological stress responses in a laboratory setting. Neuropsychobiology, 28, 76-81.

Konnai, R., Scherer, R. C., Peplinski, A., \& Ryan, K. (2017). Whisper and phonation: Aerodynamic comparisons across adduction and loudness. Journal of Voice, 31(6), 773 e711-773 e720. https://doi. org/10.1016/j.jvoice.2017.02.016.

Kryshtopava, M., Van Lierde, K., Meerschman, I., D'Haeseleer, E., Vandemaele, P., Vingerhoets, G., \& Claeys, S. (2017). Brain activity during phonation in women with muscle tension dysphonia: An fMRI study. Journal of Voice, 31, 675-690. https://doi.org/10. 1016/j.jvoice.2017.03.010.

Lorberbaum, J. P., Kose, S., Johnson, M. R., Arana, G. W., Sullivan, L. K., Hamner, M. B., . . . George, M. S. (2004). Neural correlates of speech anticipatory anxiety in generalized social phobia. Brain Imaging, 15(18), 2701-2705.

Loucks, T. M., Poletto, C. J., Simonyan, K., Reynolds, C. L., \& Ludlow, C. L. (2007). Human brain activation during phonation and exhalation: Common volitional control for two upper airway functions. Neuroimage, 36(1), 131-143.

Ludlow, C. L. (2005). Central nervous system control of the laryngeal muscles in humans. Respiratory Physiology \& Neurobiology, 147(2-3), 205-222. https://doi.org/10.1016/j.resp.2005.04.015.

Ludlow, C. L., Loucks, T., Simonyan, K., \& Lowell, S. (2008). Brain imaging of voice, swallow, and other upper airway functions. In R. J. Ingham (Ed.), Neuroimaging in communication sciences and disorders (pp. 87-127). San Diego: Plural.

MacArthur Research Network on Socioeconomic Status and Health. (2000). Salivary cortisol and challenge tests. Retrieved 01/20/ 2006, from http://www.macses.ucsf.edu/research/allostatic/ challenge.php.

McNaughton, N., \& Corr, P. J. (2004). A two-dimensional neuropsychology of defense: Fear/anxiety and defensive distance. Neuroscience and Biobehavioral Reviews, 28(3), 285-305. https://doi.org/10. 1016/j.neubiorev.2004.03.00.

Nachev, P., Kennard, C., \& Husain, M. (2008). Functional role of the supplementary and pre-supplementary motor areas. Nature Reviews. Neuroscience, 9(11), 856-869. https://doi.org/10.1038/ nrn2478.

Olthoff, A., Baudewig, J., Kruse, E., \& Dechent, P. (2008). Cortical sensorimotor control in vocalization: A functional magnetic resonance imaging study. Laryngoscope, 118(11), 2091-2096. https:// doi.org/10.1097/MLG.0b013e31817fd40f.

Patrick, C. J., Curtin, J. J., \& Tellegen, A. (2002). Development and validation of a brief form of the Multidimensional Personality Questionnaire. Psychological Assessment, 14(2), 150-163. https:// doi.org/10.1037/1040-3590.14.2.150.

Paul, G. L. (1966). Insight versus desensitization in psychotherapy: An experiment in anxiety reduction. Palo Alto, CA: Stanford University Press. 
Paus, T. (2001). Primate anterior cingulate cortex: Where motor control, drive and cognition interface. Nature Reviews. Neuroscience, 2(6), 417-424. https://doi.org/10.1038/35077500.

Paus, T., Petrides, M., Evans, A. C., \& Meyer, E. (1993). Role of the human anterior cingulate cortex in the control of oculomotor, manual, and speech responses: A positron emission tomography study. Journal of Neurophysiology, 70(2), 453-469.

Perrachione, T. K., \& Ghosh, S. S. (2013). Optimized design and analysis of sparse-sampling fMRI experiments. Frontiers in Neuroscience, 7, 55. https://doi.org/10.3389/fnins.2013.00055.

Picard, N., \& Strick, P. L. (2001). Imaging the premotor areas. Current Opinion in Neurobiology, 11(6), 663-672.

Pruessner, J. C., Kirschbaum, C., Meinlschmid, G., \& Hellhammer, D. H. (2003). Two formulas for computation of the area under the curve represent measures of total hormone concentration versus timedependent change. Psychoneuroendocrinology, 28, 916-931.

Pruessner, J. C., Dedovic, K., Khalili-Mahani, N., Engert, V., Pruessner, M., Buss, C., . . . Lupien, S. (2008). Deactivation of the limbic system during acute psychosocial stress: Evidence from positron emission tomography and functional magnetic resonance imaging studies. Biological Psychiatry, 63(2), 234-240. https://doi.org/10. 1016/j.biopsych.2007.04.041.

Rosenberg, M. (1989). Society and the adolescent self-image (revised edition). Middletown, CT: Wesleyan University Press.

Roy, N. (2003). Functional dysphonia. Current Opinion in Otolaryngology \& Head and Neck Surgery, 11(3), 144-148.

Roy, N., \& Bless, D. M. (2000). Toward a theory of the dispositional bases of functional dysphonia and vocal nodules: Exploring the role of personality and emotional adjustment. In R. D. Kent \& M. J. Ball (Eds.), Voice quality measurement (pp. 461-480). San Diego: Singular.

Roy, N., Merrill, R. M., Thibeault, S., Gray, S. D., \& Smith, E. M. (2004). Voice disorders in teachers and the general population: Effects on work performance, attendance, and future career choices. Journal of Speech, Language, and Hearing Research, 47(3), 542-551. https:// doi.org/10.1044/1092-4388(2004/042).

Roy, N., Dietrich, M., Blomgren, M., Heller, A., Houtz, D. R., \& Lee, J. (2017). Exploring the neural bases of primary muscle tension dysphonia: A case study using functional magnetic resonance imaging. Journal of Voice, 33, 183-194. https://doi.org/10.1016/j.jvoice. 2017.11.009.

Schulz, G. M., Varga, M., Jeffires, K., Ludlow, C. L., \& Braun, A. R. (2005). Functional neuroanatomy of human vocalization: $\mathrm{An}_{2}{ }^{15} \mathrm{O}$ PET study. Cerebral Cortex, 15(12), 1835-1847.

Shapiro, D., Jamner, L. D., Lane, J. D., Light, K. C., Myrtek, M., Sawada, Y., \& Steptoe, A. (1996). Blood pressure publication guidelines. Psychophysiology, 33(1), 1-12. https://doi.org/10.1111/j.14698986.1996.tb02103.x.
Simonyan, K. (2014). The laryngeal motor cortex: Its organization and connectivity. Current Opinion in Neurobiology, 28c, 15-21. https:// doi.org/10.1016/j.conb.2014.05.006.

Simonyan, K., \& Fuertinger, S. (2015). Speech networks at rest and in action: Interactions between functional brain networks controlling speech production. Journal of Neurophysiology, 113(7), 2967-2978. https://doi.org/10.1152/jn.00964.2014.

Simonyan, K., \& Horwitz, B. (2011). Laryngeal motor cortex and control of speech in humans. Neuroscientist, 17(2), 197-208. https://doi. org/10.1177/1073858410386727.

Simonyan, K., Ostuni, J., Ludlow, C. L., \& Horwitz, B. (2009). Functional but not structural networks of the human laryngeal motor cortex show left hemispheric lateralization during syllable but not breathing production. Journal of Neuroscience, 29(47), 1491214923. https://doi.org/10.1523/JNEUROSCI.4897-09.2009.

Solomon, N. P., \& Helou, L. B. (2013). Aerodynamic assessment of phonation: Avoiding common mistakes. In R. Scherer \& K. Verdolini Abbott (Eds.), The continuing influence of Ingo R. Titze on voice, science, and music: A Festschrift collection (pp. 49-56). Salt Lake City, Utah: National Center for Voice and Speech, University of Utah.

Spengler, F. B., Becker, B., Kendrick, K. M., Conrad, R., Hurlemann, R., $\&$ Schade, G. (2017). Emotional dysregulation in psychogenic voice loss. Psychotherapy and Psychosomatics, 86(2), 121-123. https:// doi.org/10.1159/000452306.

Verdolini, K., Rosen, C. A., \& Branski, R. C. (2006). Classification manual for voice disorders-1. Mahwah, NJ: Lawrence Erlbaum Associates.

Vogt, B. A. (2016). Midcingulate cortex: Structure, connections, homologies, functions and diseases. Journal of Chemical Neuroanatomy, 74, 28-46. https://doi.org/10.1016/j.jchemneu.2016.01.010.

Wager, T. D., van Ast, V. A., Hughes, B. L., Davidson, M. L., Lindquist, M. A., \& Ochsner, K. N. (2009). Brain mediators of cardiovascular responses to social threat, part II: Prefrontal-subcortical pathways and relationship with anxiety. Neuroimage, 47(3), 836-851.

Watson, D., \& Clark, L. A. (1994). The PANAS-X: Manual for the Positive and Negative Affect Schedule - Expanded Form. The University of Iowa.

Zraick, R. I., Smith-Olinde, L., \& Shotts, L. L. (2012). Adult normative data for the KayPentax Phonatory Aerodynamic System Model $6600 \ldots$ [corrected] [published erratum appears in J Voice 2013; 27(1) 2. Journal of Voice, 26(2), 164-176 113p. https://doi.org/10. 1016/j.jvoice.2011.01.006

Publisher's note Springer Nature remains neutral with regard to jurisdictional claims in published maps and institutional affiliations. 